\title{
Remarks on the Isomorphism Theorems for Weak Bernoulli Transformations in General Case
}

By

\author{
S. Ito*, H. Murata** and H. Totoki
}

\section{$\S 1 . \quad$ Introduction}

Recently D.S. Ornstein [4] proved that entropy is a complete invariant for Bernoulli shifts with finite generators. M. Smorodinsky [7] generalized this important result to the case of Bernoulli shifts with countable generators. Ornstein [5] also proved the above fact for Bernoulli shifts with infinite entropy. On the other hand N.A. Friedman and Ornstein [2] obtained the result that a weak Bernoulli transformation with finite generator is isomorphic to a Bernoulli shift with the same entropy, so that entropy is also a complete invariant for these transformations.

Moreover Ornstein [5] proved that if $T$ is a mixing (or only ergodic) transformation on a $\sigma$-field $\mathscr{F}$ and $\mathscr{F}$ is an increasing union of invariant sub- $\sigma$-fields $\mathscr{F}_{i}$ such that each $T \mid \mathscr{F}_{i}$ (restriction to $\mathscr{F}_{i}$ ) is a Bernoulli shift with finite entropy, then $T$ is itself a (generalized) Bernoulli shift. Combining this theorem and the results mentioned above, the isomorphism theorem for generalized weak Bernoulli transformation (see Definition 1 in $\S 2$ ) is easily obtained, because these transformations restricted to each approximating $\sigma$-fields are weak Bernoulli transformations with finite generators and hence Bernoulli shifts. This argument, however, make use of the results of $[2],[4],[5]$ and $[7]$. Therefore it seems to be significant to prove the isomorphism theorem in a straight way,

Received June 29, 1971.

* Department of Applied Mathematical Science, Tokyo University of Education, Otsuka Tokyo, 112, Japan.

** Department of Mathematics, Hiroshima University, Hiroshima, 730, Japan. 
unifying the above mentioned results.

The purpose of this note is to give a direct proof of the isomorphism theorem for generalized weak Bernoulli transformations. Another proof of this theorem was also obtained by G. Maruyama, which appears in this issue, from another view point using a different way.

The proof of the theorem will be divided into two steps. Firstly we will prove the theorem for weak Bernoulli transformations with countable generators having finite entropy (Proposition 1) in $\S 3$. We do this without using the isomorphism theorem for Bernoulli shifts. So this includes the results of $[4],[7]$ and $[2]$, since Bernoulli shifts are weak Bernoulli as a matter of course. Next in $\S 4$ we will prove the theorem in general case, which includes the results of $[5]$. For this purpose we use the results in §3, especially Lemma 11 which is a stronger form of Lemma 7 of [4]. Although the method of our proof is analogous to the ones in [2] and [5], we will go into details to serve the purpose of this note.

In §5 we will give some examples of weak Bernoulli transformations which contain mixing Markov shifts with countable generators. $\S 2$ is the preliminary one where we will prepare notations and definitions, especially the definition of generalized weak Bernoulli transformations.

We want to express our thanks to Professor D.S. Ornstein who kindly sent us preprints of his papers.

\section{§2. Notations and Definitions}

Let $(X, \mathscr{F}, m)$ be a non-atomic Lebesgue probability space. ${ }^{1)}$ Transformation $T$ is always invertible and measure preserving. Let $P, Q, R, \ldots$ denote measurable partitions of $X$, and when they are at most countable, we always assume they are ordered partitions. We will define some notations for (ordered) partitions. Given a partition $P=\left\{p_{1}, p_{2} \ldots\right\}, N(P)$ denotes the number of the atoms $p_{1}, p_{2}, \cdots$ of $P$ and

$$
d(P)=\left\{m\left(p_{1}\right), m\left(p_{2}\right), \ldots\right\}
$$

1) cf. [6] for the notions of the Lebesgue space and measurable partitions. 
is the probability vector of the atoms of $P$. If $N(P)>N(Q)$ then we add ideal atoms $\varnothing$ to $Q$ to make $N(P)=N(Q)$, and then we define

$$
\begin{aligned}
& d(P, Q)=\sum_{j}\left|m\left(p_{j}\right)-m\left(q_{j}\right)\right|, \\
& D(P, Q)=\sum_{j} m\left(p_{j} \triangle q_{j}\right) .
\end{aligned}
$$

Given a set $F$ with positive measure and partitions $P$ and $Q$, we will write

$$
\begin{aligned}
& m(A \mid F)=m(A \cap F) / m(F), A \in \mathscr{F}, \\
& P / F=\left\{p_{1} \cap F, p_{2} \cap F, \ldots\right\}, \\
& D(P, Q \mid F)=D(P / F, Q / F)=\sum_{j} m\left(p_{j} \triangle q_{j} \mid F\right) .
\end{aligned}
$$

Given partitions $P$ and $Q, Q \mathcal{C}^{\varepsilon} P$ denotes that there exists a partition $\bar{P}$ such that $P$ is a refinement of $\bar{P}$ and $D(\bar{P}, Q)<\varepsilon$. We write $Q \subset P$ if $D(\bar{P}, Q)=0$. Note that $\bar{P}$ can be realized in the following way. Consider a partition $L=\left\{l_{1}, l_{2}, \ldots\right\}$ of the index set $\{1,2, \ldots, N(P)\}$ of $P$. Then we can define a partition $L_{P}$ of $X$ as $L_{P}=\left\{\bigcup_{i \in l_{j}} p_{i}, j=1,2, \ldots\right\}$; hence $N\left(L_{P}\right)=N(L)$. Thus there is a partition $L$ such that $L_{P}=\bar{P}$. If $Q$ is a subfamily of the sub- $\sigma$-field generated by $P$, then we will also write $Q \subset P$. If $P$ and $Q$ are partitions of $X, P \vee Q, \vee_{0}^{n} T^{i} P, \ldots$ will denote the ordered partitions with a canonical ordering.

Now we define several entropies as usual. Given countable partitions $P$ and $Q$, we have the following

$$
\begin{aligned}
& H(P)=-\sum_{j} m\left(p_{j}\right) \log m\left(p_{j}\right), \\
& H(P \mid Q)=-\sum_{i, j} m\left(q_{i}\right) m\left(p_{j} \mid q_{i}\right) \log m\left(p_{j} \mid q_{i}\right), \\
& h(P, T)=\lim _{n \rightarrow \infty} \frac{1}{n} H\left(P \vee \cdots \vee T^{n-1} P\right) \\
& h(T)=\sup _{P} h(P, T)
\end{aligned}
$$

where the supremum is taken over all finite partitions of $X$. Note that 
$H\left(P \mid \bigvee_{-n}^{-1} T^{i} P\right)$ decreases to $h(P, T)$

We will remark the following. Let $\mathscr{P}$ and $\mathscr{P}_{k}$ denote the collections of all countable partitions and partitions with at most $k$ atoms respectively. Firstly, $(\mathscr{P}, d)$ and $(\mathscr{P}, D)$ are complete separable metric spaces. Next, the entropy $H(P)$ is continuous in $\left(\mathscr{P}_{k}, d\right)$ and $\left(\mathscr{P}_{k}, D\right)$. At last, the entropy $h(\bullet, T)$ is continuous in $\left(\mathscr{P}_{k}, D\right)$, and it is only lower semicontinuous in $(\mathscr{P}, D)$. These properties will be used in the proof of the theorem.

Given countable partitions $P$ and $Q$, we say $P$ and $Q$ are $\varepsilon$-independent and denote $P \perp \mathcal{\varepsilon}$ if

$$
\sum_{i, j}\left|m\left(p_{i} \cap q_{j}\right)-m\left(p_{i}\right) m\left(q_{j}\right)\right|<\varepsilon .
$$

Although this definition is different from the one in [4], it is easy to see that they are equivalent, and our definition is convenient in the point that if $P \stackrel{\varepsilon}{\perp} Q$ and $\bar{P} \subset P, \bar{Q} \subset Q$ then $\bar{P} \perp \bar{Q}$.

Definition 1. A countable partition $P$ is called weak Bernoulli for $T$ if for each $\varepsilon>0$ there exists $K=K(\varepsilon, P)$ such that $\bigvee_{-n}^{0} T^{i} P \perp{ }_{K}^{\varepsilon+n} T^{i} P$ for every $n \geqq 0$. A measurable partition $P$ is called generalized ${ }^{K}$ weak Bernoulli for $T$ if there exists an increasing sequence $\left\{P_{n}\right\}$ of finite partitions such that $\vee_{1}^{\infty} P_{n}=P$ and each $P_{n}$ is weak Bernoulli for $T$. In this case the invariant sub- $\sigma$-fields generated by $\underset{-\infty<i<\infty}{\vee} T^{i} P_{n}(n \geqq 1)$ are called approximating $\sigma$-fields. The transformation $T$ is called (generalized) weak Bernoulli if $T$ has a (generalized) weak Bernoulli generator.

\section{§3. Countable Case}

In this section we will prove the following

Proposition 1. Two weak Bernoulli transformations with the same entropy whose weak Bernoulli generators are at most countable and have finite entropies are isomorphic.

For the proof of this proposition, we prepare some lemmas in which 
we are concerned only with countable partitions.

Lemma 1. Let $\varepsilon>0$ and let $Q_{i}, Q_{i}^{\prime}, 0 \leqq i \leqq n, P_{0}, P_{0}^{\prime}$ be partitions such that

$$
\begin{gathered}
Q_{0} \perp \stackrel{\varepsilon}{\vee}_{1}^{n} Q_{i}, \\
Q_{0}^{\prime} \perp \stackrel{n}{\vee}_{1}^{*} Q_{i}^{\prime}, \\
d\left(\bigvee_{1}^{n} Q_{i}, \stackrel{n}{\vee} Q_{i}^{\prime}\right)<\varepsilon, \\
d\left(Q_{0}\right)=d\left(P_{0}\right), d\left(Q_{0}^{\prime}\right)=d\left(P_{0}^{\prime}\right) .
\end{gathered}
$$

Then there exist partitions $P_{i}, P_{i}^{\prime}, 1 \leqq i \leqq n$, such that

$$
\begin{gathered}
d\left(\bigvee_{0}^{n} Q_{i}\right)= \\
d\left(\bigvee_{0}^{n} P_{i}\right), \quad d\left(\bigvee_{0}^{n} Q_{i}^{\prime}\right)=d\left(\bigvee_{0}^{n} P_{i}^{\prime}\right), \\
\sum_{i=1}^{n} D\left(P_{i}, P_{i}^{\prime}\right)<3 n \varepsilon .
\end{gathered}
$$

Noting the difference of the definitions of $\varepsilon$-independence, this lemma is the same as Lemma 3 in [2], so we may omit the proof.

Similarly the following lemma is the same as Lemma 1 in [7], so we also omit the proof.

Lemma 2. For each $\varepsilon>0$ there exists $\zeta=\zeta(\varepsilon)>0$ such that if the partitions $P$ and $Q$ satisfy

$$
H(P)-H(P \mid Q)<\zeta,
$$

then $P \stackrel{\varepsilon}{\perp} Q$

Using this lemma we can prove the following

Lemma 3. Let $\varepsilon>0$ and $\zeta=\zeta(\varepsilon)>0$ as in Lemma 2. If $P_{0}, P, Q$ and $R$ are partitions such that

$$
P_{0} \subset P
$$




$$
\begin{gathered}
P_{0} \stackrel{\varepsilon}{\perp} Q, \\
H(P \mid Q)-H(P \mid Q \vee R)<\varepsilon \zeta,
\end{gathered}
$$

then

$$
P_{0} \stackrel{4}{\perp} Q \vee R
$$

Proof. Note that

$$
H(P \mid Q)-H(P \mid Q \vee R)=\sum_{q \in Q} m(q)\{H(P / q)-H(P / q \mid R / q)\}
$$

Put $Q_{1}=\{q \in Q ; H(P / q)-H(P / q \mid R / q)<\zeta\}$. Because of (3.3), we have $m\left(\cup Q_{1}\right)>1-\varepsilon$. Lemma 2 implies $P / q \perp R / q$ for all $q \in Q_{1}$. Therefore

$$
\begin{aligned}
& \sum_{p \in P_{0}, q \in Q, r \in R}|m(p \cap q \cap r)-m(p) m(q \cap r)| \\
& \leqq \sum_{p, q, r} m(q)\{|m(p \cap r \mid q)-m(p \mid q) m(r \mid q)| \\
& \quad+m(r \mid q)|m(p \mid q)-m(p)|\} \\
& =\sum_{q \in Q_{1}} m(q) \sum_{p, r}|m(p \cap r \mid q)-m(p \mid q) m(r \mid q)| \\
& \quad+\sum_{q \notin Q_{1}} m(q) \sum_{p, r}|m(p \cap r \mid q)-m(p \mid q) m(r \mid q)| \\
& \quad+\sum_{p, q}|m(p \cap q)-m(p) m(q)| \sum_{r} m(r \mid q)
\end{aligned}
$$

$<4 \varepsilon$.

As we remarked in $\S 2$, the entropy of countable partitions is not continuous in $d$-metric, we have to add one more condition on the entropy to Lemmas 8,9 and so on in [2].

Lemma 4. Let $P$ be weak Bernoulli for $T$ with $H(P)<\infty$, and let $\varepsilon>0$. Let $K=K(\varepsilon / 4, P)$ be as in Definition 1. Let $H$ be a positive integer. Then there exist a positive integer $n_{*}$ and $\eta>0$ such that if $T^{\prime}$ and $P^{\prime}$ satisfy

$$
d\left(\bigvee_{-n_{*}}^{K+H} T^{i} P, \quad \bigvee_{-n_{*}}^{K+H} T^{\prime^{i}} P^{\prime}\right)<\eta
$$




$$
\begin{gathered}
\left|h(P, T)-h\left(P^{\prime}, T^{\prime}\right)\right|<\eta, \\
\left|H\left(\bigvee_{-n_{*}}^{j} T^{i} P\right)-H\left(\bigvee_{-n_{*}}^{j} T^{\prime i} P^{\prime}\right)\right|<\eta, \quad-n_{*} \leqq j \leqq K+H,
\end{gathered}
$$

then for each $m \geqq 0$,

$$
\bigvee_{K}^{K+H} T^{\prime i} P^{\prime} \stackrel{\varepsilon}{\perp} \underset{-m}{\vee} T^{\prime i} P^{\prime}
$$

Proof. Let $P_{1}=\bigvee_{1}^{K+H} T^{i} P, P_{2}=\bigvee_{K}^{K+H} T^{i} P, Q=\bigvee_{-n}^{0} T^{i} P$, and $R=\bigvee_{-n-m}^{-n-1} T^{i} P$. We define $P_{1}^{\prime}, P_{2}^{\prime}, Q^{\prime}$, and $R^{\prime}$ in the same way for $T^{\prime}$ and $P^{\prime}$. Since $H\left(P_{1} \mid Q\right)$ decreases monotonically to $H\left(P_{1} \mid \stackrel{0}{\vee} T^{i} P\right)=(K+H) h(P, T)$ as $n \rightarrow \infty$, we can take $n_{*}=n$ so large that

(1) $(K+H) h(P, T)+\varepsilon \zeta / 4-H\left(P_{1} \mid Q\right)=\alpha>0$,

where $\zeta=\zeta(\varepsilon / 4)$ is as in Lemma 2. The choice of $K$ implies

(2) $P_{2} \stackrel{\varepsilon / 4}{\perp} Q$.

Now take $\eta>0$ so small that (2) and (4.1) imply

(3) $P_{2}^{\prime} \stackrel{\varepsilon / 4}{\perp} Q^{\prime}$,

(4.2) implies

(4) $\left|(K+H) h(P, T)-(K+H) h\left(P^{\prime}, T^{\prime}\right)\right|<\alpha / 2$, and (4.3) implies

(5) $\left|H\left(P_{1} \mid Q\right)-H\left(P_{1}^{\prime} \mid Q^{\prime}\right)\right|<\alpha / 2$.

Then (1), (4) and (5) imply

(6) $H\left(P_{1}^{\prime} \mid Q^{\prime}\right)<H\left(P_{1}^{\prime} \mid Q^{\prime} \vee R^{\prime}\right)+\varepsilon \zeta / 4$

Therefore (3) and (6) imply (4.4) by Lemma 3 .

Lemma 5. Let $P$ be weak Bernoulli for $T$ with $H(P)<\infty$ and let $\varepsilon>0$. There exist positive integers $n_{1}, n_{2}$, and $\eta>0$ such that if $T^{\prime}$ and $P^{\prime}$ satisfy 


$$
\begin{gathered}
d\left(\bigvee_{0}^{n_{1}} T^{i} P, \bigvee_{0}^{n_{1}} T^{\prime i} P^{\prime}\right)<\eta, \\
\left|h(P, T)-h\left(P^{\prime}, T^{\prime}\right)\right|<\eta, \\
\left|H\left(\bigvee_{0}^{j} T^{i} P\right)-H\left(\bigvee_{0}^{j} T^{\prime i} P^{\prime}\right)\right|<\eta, \quad 0 \leqq j \leqq n_{1},
\end{gathered}
$$

then there exist partitions $P_{i}$ and $P_{i}^{\prime}$ of $X$ such that

$$
\begin{array}{cl}
d\left(\bigvee_{0} P_{i}\right)=d\left(\bigvee_{0}^{\vee} T^{i} P\right), & n=0,1,2, \ldots, \\
d\left(\bigvee_{0}^{n} P_{i}^{\prime}\right)=d\left(\bigvee_{0}^{n} T^{\prime i} P^{\prime}\right), & n=0,1,2, \ldots, \\
& \sum_{i=0}^{n} D\left(P_{i}, P_{i}^{\prime}\right)<\varepsilon n, \quad n \geqq n_{2} .
\end{array}
$$

Proof. Let $K=K(\varepsilon / 50, P)$ be as in Definition 1 . Choose $H$ so that $K / K+H<\varepsilon / 8$. Apply Lemma 4 to $H, K$ and $\varepsilon / 12$, then we have $n_{*}$ and $\eta<\varepsilon / 12$. Let $n_{1}=n_{*}+K+H$. The choice of $K$ and Lemma 4 implies

$$
\bigvee_{K+n}^{K+H} T^{i} P \stackrel{\varepsilon / 12}{\perp} \bigvee_{0}^{n} T^{i} P, \underset{K+n}{\bigvee^{+H+n}} T^{{ }^{i}} P^{\prime} \stackrel{\varepsilon / 12}{\perp} \bigvee_{0}^{n} T^{\prime i} P^{\prime}
$$

for all $n \geqq 0$.

We will define $P_{i}, P_{i}^{\prime}$ inductively. Let $P_{0}$ and $P_{0}^{\prime}$ be partitions of $X$ such that

(2) $\quad d\left(P_{0}\right)=d(P), d\left(P_{0}^{\prime}\right)=d\left(P^{\prime}\right)$.

Let $Q_{0}=P, Q_{0}^{\prime}=P^{\prime}$, and

(3) $Q_{i}=T^{i+K-1} P, Q_{i}^{\prime}={T^{\prime 2+K-1}}^{\prime}, \quad 1 \leqq i \leqq K+H$.

Because of (1) (in case of $n=0),(2)$ and (5.1) we can apply Lemma 1 to get $P_{i}, P_{i}^{\prime}, K \leqq i \leqq K+H$ such that
(4) $\quad d\left(P_{0} \vee \bigvee_{K}^{K+H} P_{i}\right)=d\left(P \vee \bigvee_{K}^{K+H} T^{i} P\right)$,
(5) $\quad d\left(P_{0}^{\prime} \vee \bigvee_{K}^{K+H} P_{i}^{\prime}\right)=d\left(P^{\prime} \bigvee \bigvee_{K}^{K+H} T^{\prime i} P^{\prime}\right)$,
(6) $\sum_{i=K}^{K+H} D\left(P_{i}, P_{i}^{\prime}\right)<3(H+1) \varepsilon / 12=(H+1) \varepsilon / 4$.

We may now define $P_{i}$ and $P_{i}^{\prime}, 1 \leqq i \leqq K-1$, so that (5.4) and (5.5) hold 
for $n \leqq K+H$. Now (6) implies

$$
\begin{aligned}
& \sum_{i=0}^{K+H} D\left(P_{i}, P_{i}^{\prime}\right)<\sum_{i=0}^{K-1} D\left(P_{i}, P_{i}^{\prime}\right)+(H+1) \varepsilon / 4 \\
& \leqq 2 K+(H+1) \varepsilon / 4<(K+H) \varepsilon / 2 .
\end{aligned}
$$

Let $n_{2}=K+H$.

Assume we have already defined $P_{i}$ and $P_{i}^{\prime}, 0 \leqq i \leqq j(K+H)$, so that (5.4-6) hold. Assume also

(7) $\sum_{t(K+H)-H}^{t(K+H)} D\left(P_{i}, P_{i}^{\prime}\right)<(H+1) \varepsilon / 4, \quad 1 \leqq t \leqq j$.

By (5.1) we have

(8) $\quad d\left(\bigvee_{n+K}^{n+K+H} T^{i} P, \bigvee_{n+K}^{n+K+H} T^{\prime i} P^{\prime}\right)<\varepsilon / 12$.

Let $n_{0}=j(K+H)$. Because of (1), (5) and (5.4-6) (for $n=j(K+H)$ ) we can apply Lemma $1\left(P_{0}\right.$ and $P_{0}^{\prime}$ are replaced by $\bigvee_{0}^{n_{0}} P_{i}$ and $\bigvee_{0}^{n_{0}} P_{i}^{\prime}$ respectively) to get $P_{i}, P_{i}^{\prime}, n_{0}+K \leqq i \leqq n_{0}+K+H$, such that

(9) $\quad d\left(\bigvee_{0}^{n_{0}} P_{i} \vee \bigvee_{n_{0}+K}^{n_{0}+K+H} P_{i}\right)=d\left(\bigvee_{0}^{n_{0}} T^{i} P \bigvee \bigvee_{n_{0}+K}^{n_{0}+K+H} T^{i} P\right)$,

$$
\begin{aligned}
& d\left(\bigvee_{0}^{n_{0}} P_{i}^{\prime} \bigvee \bigvee_{n_{0}+K}^{n_{0}+K+H} P_{i}^{\prime}\right)=d\left(\bigvee_{0}^{n_{0}} T^{\prime 2} P^{\prime} \bigvee{ }_{n_{0}+K}^{n_{0}+K+H} T^{\prime 2} P^{\prime}\right), \\
& \sum_{i=n_{0}+K}^{n_{0}+K+H} D\left(P_{i}, P_{i}^{\prime}\right)<(H+1) \varepsilon / 4
\end{aligned}
$$

We define $P_{i}, P_{i}^{\prime}, n_{0}<i<n_{0}+K$, so that (5.4) and (5.5) hold for $n=(j+1)(K+H)$. For $j(K+H)<n \leqq(j+1)(K+H)$, (7) and (11) imply

$$
\begin{aligned}
\sum_{i=0}^{n} D\left(P_{i}, P_{i}^{\prime}\right) & <2 K j+j(H+1) \varepsilon / 4+2 K+(H+1) \varepsilon / 4 \\
& <n \varepsilon .
\end{aligned}
$$

We will now prepare the following two lemmas which will be used to prove the approximation lemma (Lemma 8). Since they are exactly the same as Lemma 10 and 11 in [2], we will omit their proofs.

We consider a transformation $T_{1}$ and a finite partition $R=\left\{r_{i} ; 1 \leqq\right.$ 
$i \leqq k\}$. Let $Q=\bigvee_{0}^{u} T_{1}^{i} R$. Note that an atom $q \in Q$ has the form

$$
q=\bigcap_{0}^{u} T_{1}^{i} r_{s_{2}}, \quad 1 \leqq s_{i} \leqq k, \quad 0 \leqq i \leqq u .
$$

Let $l=\left\{l_{0}, l_{1}, \ldots, l_{n-1}\right\}$ be a sequence of length $n(>u)$, where $1 \leqq l_{i} \leqq$ $k, 0 \leqq i<n$. Let $N(l, q)$ be the number of times that $\left(s_{u}, s_{u-1}, \cdots, s_{0}\right)$ appears as a consecutive subsequence in $l$; hence $N(l, q) \leqq n-u$.

Definition 2. Let $\varepsilon>0 . l$ is called an $\varepsilon$-sequence for $Q$ if

$$
|N(l, q) / n-m(q)|<\varepsilon
$$

for all $q \in Q$.

Lemma 6. Let $R, T_{1}$, and $Q$ be as above and $\varepsilon>0$. Let $T$ be a transformation and let $B_{j}, 1 \leqq j \leqq J$, be measurable sets such that $T^{i} B_{j}$, $0 \leqq i<n, 1 \leqq j \leqq J$, are disjoint. Assume $u / n<\varepsilon / 4$ and $X_{1}=\bigcup_{i=0}^{n-1} \bigcup_{j=1}^{J} T^{i} B_{j}$ satisfies $m\left(X_{1}\right)>1-\varepsilon / 4$. Let $l_{j}=\left\{l_{j, i}, 0 \leqq i<n\right\}, 1 \leqq j \leqq J$, be $\varepsilon / 4 k^{u+1}$ -sequences for $Q$. Let $P=\left\{p_{1}, \cdots, p_{k}\right\}$ be a partition such that $p_{t} / X_{1}=$ $\bigcup_{l_{j}=t} T^{i} B_{j}$ and $p_{t} / X_{1}^{c}$ is arbitrary for $1 \leqq t \leqq k$. Then

$$
d\left(\stackrel{u}{\vee}_{0}^{i} T^{i} P, Q\right)<\varepsilon .
$$

If the sequences $l_{j}, 1 \leqq j \leqq J$, are distinct, then

$$
\bigvee_{-n}^{n} T^{i}\left(P \vee\left\{B, B^{c}\right\}\right) \supset\left\{T^{i} B_{j}: 0 \leqq i<n, 1 \leqq j \leqq J\right\}
$$

where $B=\bigcup_{j=1}^{J} B_{j}$.

Lemma 7. Let $T_{1}$ be ergodic. Let $R=\left\{r_{1}, \ldots, r_{k}\right\}$ be a finite partition and $Q=\bigvee_{0}^{u} T_{1}^{i} R$. Let $L_{n}=\bigvee_{0}^{n-1} T_{1}^{-i} R$ and write $l=\bigcap_{0}^{n-1} T_{1}^{-i} r_{l_{i}} \in L_{n}$ as $l=\left(l_{0}, l_{1}, \ldots, l_{n-1}^{0}\right)$. Let $a>0$ and $b>0$ be arbitrary. Then there exists $n_{0}$ such that for all $n \geqq n_{0}$ we have a subfamily $L_{n}^{\prime} \subset L_{n}$ such that $m\left(\cup L_{n}^{\prime}\right)>1-a$, and $l \in L_{n}^{\prime}$ implies $l$ is a b-sequence for $Q$.

Now we can prove the following approximation lemma which is 
different from the Lemma 12 in $[2]$. Precisely speaking, the condition that $T$ is "Bernoulli" in [2] is only used in changing the partition $P$ to satisfy the condition $0<h\left(T_{1}\right)-h(P, T)<\delta$ in the case $h\left(T_{1}\right)=h(P, T)$. Noting this point, we can state this lemma in the following way.

Lemma 8. Let $R$ be weak Bernoulli for $T_{1}$ with $h\left(R, T_{1}\right)=h\left(T_{1}\right)$ and $H(R)<\infty$. Let $n_{1}, n_{2}$, and $\eta$ be as in Lemma 5 for $R, T_{1}$, and $\varepsilon^{2} / 3$. Let $T$ be an ergodic transformation with $h(T)=h\left(T_{1}\right)$. Let $P^{\prime}$ be a partition such that

$$
\begin{gathered}
d\left(\bigvee_{0}^{n_{1}} T_{1}^{i} R, \bigvee_{0}^{n_{1}} T^{i} P^{\prime}\right)<\eta, \\
h\left(T_{1}\right)-h\left(P^{\prime}, T\right)<\eta, \\
\left|H\left(\bigvee_{0}^{j} T_{1}^{i} R\right)-H\left(\bigvee_{0}^{j} T^{i} P^{\prime}\right)\right|<\eta, \quad 0 \leqq j \leqq n_{1} .
\end{gathered}
$$

Then given $\delta>0$ and a positive integer $u$, there exists a (finite) partition $P$ such that

$$
\begin{gathered}
d\left(\bigvee_{0}^{u} T_{1}^{i} R,{\underset{0}{\vee}}_{0}^{i} T^{i} P\right)<\delta, \\
h\left(T_{1}\right)-h(P, T)<\delta, \\
\left|H\left(\bigvee_{0}^{j} T_{1}^{i} R\right)-H\left(\bigvee_{0}^{j} T^{i} P\right)\right|<\delta, \quad 0 \leqq j \leqq u, \\
D\left(P^{\prime}, P\right)<6 \varepsilon .
\end{gathered}
$$

Proof. We first assume $h\left(T_{1}\right)=h\left(R, T_{1}\right)>h\left(P^{\prime}, T\right)$. Let $R=\left\{r_{1}, r_{2}, \ldots\right\}$ and define the $k$-section $R(k)=\left\{r_{1}, r_{2}, \cdots, r_{k-1}, \bigcup_{j \geqq k} r_{j}\right\}$ of $R . P^{\prime}(k)$ is also difined in the same way. Then we can take $k$ so large that

$$
\begin{aligned}
& \text { (1) } d\left(\bigvee_{0}^{v} T_{1}^{i} R, \underset{0}{\vee} T_{1}^{i} R(k)\right)<\delta / 2, \\
& \text { (2) } H\left(\bigvee_{0}^{j} T_{1}^{i} R\right)-H\left(\bigvee_{0}^{j} T_{1}^{i} R(k)\right)<\delta / 2, \quad 0 \leqq j \leqq u, \\
& \text { (3) } h\left(T_{1}\right)-h\left(R(k), T_{1}\right)<\delta / 4, \\
& \text { (4) } h\left(P^{\prime}, T\right)<h\left(R(k), T_{1}\right),
\end{aligned}
$$


(5) $D\left(P^{\prime}, P^{\prime}(k)\right)<\varepsilon$.

Denote $\bar{R}=R(k)$, and also $\bar{P}=P^{\prime}(k)$. Take $\delta_{1}<\delta / 2$ such that if $Q_{i}, 0 \leqq$ $i \leqq u_{j}$ are partitions with $k$ atoms and if $d\left(\underset{j}{\vee} T_{1}^{i} \bar{R}, \stackrel{u}{\vee} Q_{i}\right)<\delta_{1}$ then $\left|H\left(\bigvee_{0}^{j} T_{1}^{i} \bar{R}\right)-H\left(\bigvee_{0}^{j} Q_{i}\right)\right|<\delta / 2,0 \leqq j \leqq u$. Because of $h\left(T_{1}\right)^{0}=h(T)$, there exists a finite refinement $W$ of $\bar{P}$ such that

(6) $0<h\left(\bar{R}, T_{1}\right)-h(W, T)=\beta<\delta / 4$.

Choose $r<\min \left(\delta_{1 / 2}, \varepsilon / 3,5 / 2\right)$ such that $D\left(W^{\prime}, W\right)<\gamma$ implies

$$
h\left(W^{\prime}, T\right)>h(W, T)-\delta / 4 .
$$

We will now choose $n$ so large that the following conditions (7)(10) are satisfied.

(7) Applying Lemma 7 to $T_{1}, \bar{R}$, and $Q=\bigvee_{0}^{n} T_{1}^{i} \bar{R}$, we have a subfamily $\mathscr{L}^{\prime} C L_{n}=\bigvee_{0}^{n-1} T_{1}^{-i} \bar{R}$ such that $m\left(\cup \mathscr{L}^{\prime}\right)>1-\gamma / 10$, and $l \in \mathscr{L}^{\prime}$ implies

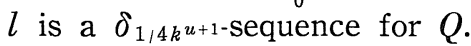

(8) Applying the Shannon-McMillan-Breiman theorem to $T_{1}$ and $\bar{R}$, we have a subfamily $\mathscr{L}^{\prime \prime} C L_{n}$ such that $m\left(\cup \mathscr{L}^{\prime \prime}\right)>1-\gamma / 10$, and $l \in \mathscr{L}^{\prime \prime}$ implies $m(l)$ is between $2^{-\left[h\left(\bar{R}, T_{1}\right) \pm \beta / 3\right] n}$.

(9) Applying the Shannon-McMillan-Breiman theorem to $T$ and $W$, we have a subfamily $\mathscr{W}_{n} \subset \bigvee_{0}^{n-1} T^{-i} W$ such that $m\left(\cup \mathscr{W}_{n}\right)>1-\gamma / 10$, and $w \in \mathscr{W}_{n}$ implies $m(w)$ is between $2^{-[h(W, T) \pm \beta / 3] n}$.

(10) $n>\max \left(n_{1}, n_{2}\right)$ such that $u / n<\delta_{1 / 4}, n \beta>3$, and $m(A) \leqq 1 / n$ implies $H\left(\left\{A, A,{ }^{c}\right\}\right)<\beta$.

Apply Lemma 5 to obtain partitions $R_{i}^{\prime}, P_{i}^{\prime}, 0 \leqq i \leqq n-1$, such that

$$
\begin{aligned}
& d\left(\bigvee_{0}^{n-1} R_{i}^{\prime}\right)=d\left(\bigvee_{0}^{n-1} T_{1}^{-i} R\right), d\left(\bigvee_{0}^{n-1} P_{i}^{\prime}\right)=d\left(\bigvee_{0}^{n-1} T^{-i} P^{\prime}\right), \\
& \sum_{i=0}^{n-1} D\left(R_{i}^{\prime}, P_{i}^{\prime}\right)<n \varepsilon^{2} / 3 .
\end{aligned}
$$

Let $\varphi$ be an automorphism of $X$ such that

$$
\varphi\left(\bigvee_{0}^{n-1} P_{i}^{\prime}\right)=\bigvee_{0}^{n-1} T^{-i} P^{\prime}
$$

Putting $R_{i}^{\prime \prime}=\varphi\left(R_{i}^{\prime}\right), 0 \leqq i \leqq n-1$, we have 


$$
d\left(\bigvee_{0}^{n-1} R_{i}^{\prime \prime}\right)=d\left(\bigvee_{0}^{n-1} T_{1}^{-i} R\right), \quad \sum_{i=0}^{n-1} D\left(R_{i}^{\prime \prime}, T^{-i} P^{\prime}\right)<n \varepsilon^{2} / 3
$$

Hence there exist partitions $R_{i}=\left\{r_{i, 1}, \ldots, r_{i, k}\right\}, 0 \leqq i \leqq n-1$, such that

(11) $\quad d\left(\bigvee_{0}^{n-1} R_{\imath}\right)=d\left(\bigvee_{0}^{n-1} T_{1}^{-i} \bar{R}\right)$

$$
\sum_{i=0}^{n-1} D\left(R_{i}, T^{-i} \bar{P}\right)<n \varepsilon^{2} / 3
$$

Let $\quad L_{n}^{*}=\bigvee_{0}^{n-1} R_{i} \quad$ and $\quad \bar{P}_{n}=\bigvee_{0}^{n-1} T^{-i} \bar{P}$. A sequence $\left(s_{0}, s_{1}, \ldots, s_{n-1}\right)$ is called the $L_{n}^{*}$-name of $x$ if $x \in \bigcap_{0}^{n-1} r_{i, s_{i}}$, and we denote this by $s^{*}(x)=$ $\left(s_{0}^{*}(x), \ldots, s_{n-1}^{*}(x)\right)$. Similarly $\left(s_{0}, s_{1}, \cdots, s_{n-1}\right)$ is called the $\bar{P}_{n}$-name of $x$ if $x \in \bigcap_{0}^{n-1} T^{-i} \bar{p}_{s_{2}}$ where $\bar{P}=\left\{\bar{p}_{1}, \cdots, \bar{p}_{k}\right\}$, and we denote this by $\bar{s}(x)=$ $\left(\bar{s}_{0}(x), \ldots, \bar{s}_{n-1}(x)\right)$. Note that for each atom $l \in L_{n}^{*} s^{*}(x)$ is the same for all $x \in l$. Hence we can talk about the $L_{n}^{*}$-name $s^{*}(l)$ of $l \in L_{n}^{*}$. Analogously we denote the $\bar{P}_{n}$-name of $w \in \bigvee_{0}^{n-1} T^{-i} W$ by $\bar{s}(w)$. Define

$$
\begin{aligned}
& \rho\left(s^{*}(x), \bar{s}(x)\right)=\text { the number of }\left\{i ; s_{i}^{*}(x) \neq \bar{s}_{i}(x)\right\} \\
& G=\left\{x ; \rho\left(s^{*}(x), \bar{s}(x)\right)>n \varepsilon\right\} .
\end{aligned}
$$

Then

$$
\begin{aligned}
n \varepsilon m(G) & \leqq E\left\{\rho\left(s^{*}(x), \bar{s}(x)\right)\right\}=\sum_{i=0}^{n-1} m\left\{x ; s_{i}^{*}(x) \neq \bar{s}_{i}(x)\right\} \\
& =\frac{1}{2} \sum_{i=0}^{n-1} D\left(R_{i}, T^{-i} \bar{P}\right)<n \varepsilon^{2} / 6
\end{aligned}
$$

and so

(13) $m(G)<\varepsilon / 6$.

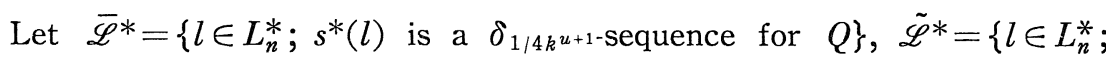
$m(l)$ is between $\left.2^{-\left[h\left(\bar{R}, T_{1}\right) \pm \beta / 3\right] n}\right\}$ and $\mathscr{L}=\overline{\mathscr{L}}^{*} \cap \tilde{\mathscr{L}}^{*}$. Then (7), (8) and (11) imply

(14) $m(\cup \mathscr{L})>1-r / 5$.

Let $\mathscr{C}=\left\{w \in \mathscr{W}_{n} ; m\left(w \cap(\cup \mathscr{L}) \cap G^{c}\right) \geqq m(w) / 2\right\}$, then 


$$
\begin{aligned}
m(X \backslash \cup \mathscr{C}) & =\sum_{w \notin \mathscr{C}} m(w) \\
& =\sum_{w \notin \mathscr{C}}\left\{m\left(w \cap(\cup \mathscr{L}) \cap G^{c}\right)+m\left(w \cap\left((\cup \mathscr{L}) \cap G^{c}\right)^{c}\right)\right\} \\
& <\frac{1}{2} \sum_{w \notin \mathscr{C}} m(w)+m(X \backslash \cup \mathscr{L})+m(G)
\end{aligned}
$$

and so

$$
\text { (15) } m(X \backslash \cup \mathscr{C})<2\{m(X \backslash \cup \mathscr{L})+m(G)\}<\varepsilon / 2
$$

Note that (6) and (10) imply

$$
\begin{aligned}
\max _{l \in \mathscr{L}} m(l) & \leqq 2^{-\left[h\left(\bar{R}, T_{1}\right)-\beta / 3\right] n} \\
& <\frac{1}{2} \cdot 2^{-[h(W, T)+\beta / 3] n} \leqq \frac{1}{2} \min _{w \in \mathscr{\mathscr { W }}} m(w) .
\end{aligned}
$$

To each $w \in \mathscr{C}$ we assign $l \in \mathscr{L}$ such that $l \cap w \cap G^{c} \neq \varnothing$. Then any $t$ elements in $\mathscr{C}$ correspond to at least $t$ elements in $\mathscr{L}$. Hence, applying the marriage lemma, each $w \in \mathscr{C}$ can be assigned an $l(w) \in \mathscr{L}$ such that $\rho\left(s^{*}(l(w)), \bar{s}(w)\right) \leqq n \varepsilon$ and the mapping $l$ is one-to-one. Since

the number of elements of $\mathscr{W}_{n}$

$$
\begin{aligned}
& \leqq 2^{\lceil h(W, T)+\beta / 3\rceil n} \\
& <(1-\gamma / 5) 2^{\left[h\left(\bar{R}, T_{1}\right)-\beta / 3\right] n} \\
& <\text { the number of elements of } \mathscr{L},
\end{aligned}
$$

we can extend the map $l$ from $\mathscr{W}_{n}$ into $\mathscr{L}$ in the one-to-one way. Thus we obtain



(17) if $w \in \mathscr{C}$ then $\rho\left(s^{*}(l(w)), \bar{s}(w)\right) \leqq n \varepsilon$.

Rohlin's theorem asserts that there exists a set $F^{\prime}$ such that $T^{i} F^{\prime}$, $0 \leqq i \leqq n-1$, are disjoint and 
(18) $m\left(\bigcup_{0}^{n-1} T^{i} F^{\prime}\right)>1-\gamma / 10$

Becaue of (15) we have

$$
\sum_{i=0}^{n-1} m\left(T^{i} F^{\prime} \cap(X \backslash \cup \mathscr{C})\right)<\varepsilon / 2 .
$$

Therefore for more than half of indices $i$,

$$
\text { (19) } m\left(T^{i} F^{\prime} \cap(X \backslash \cup \mathscr{C})\right)<\varepsilon / n
$$

Analogously using (9) we have

$$
\text { (20) } m\left(T^{i} F^{\prime} \cap\left(X \backslash \cup \mathscr{W}_{n}\right)\right)<r / 5 n
$$

for more than half of indices $i$. Hence there exists $i=i_{0}$ such that (19) and (20) hold together. Put $F=T^{i_{0}} F^{\prime}$. Then $T^{i} F, 0 \leqq i \leqq n-1$, are disjoint and

$$
\begin{aligned}
& \text { (21) } m\left(\bigcup_{0}^{n-1} T^{i}(F \cap \cup \mathscr{C})\right)>1-\gamma / 10-\varepsilon, \\
& \text { (22) } m\left(\bigcup_{0}^{n-1} T^{i}\left(F \cap \cup \mathscr{W}_{n}\right)\right)>1-3 \gamma / 10 .
\end{aligned}
$$

We will now define the partition $P=\left\{p_{1}, p_{2}, \cdots, p_{k}\right\}$ as follows. Let $X_{1}=\bigcup_{0}^{n-1} T^{i}\left(F \cap \cup \mathscr{W}_{n}\right)$ and $A=F \cap \cup \mathscr{W}_{n}$, then

$$
\text { (23) } m(A) \leqq 1 / n, \quad m\left(X_{1}\right)>1-3 r / 10>1-\delta_{1 / 4} .
$$

Define $P$ on $X_{1}$ as

$$
p_{j} / X_{1}=\cup\left\{T^{i} w ; w \in \mathscr{W}_{n} / F, s_{1}^{*}(l(w))=j\right\}, \quad 1 \leqq j \leqq k .
$$

Obviously $P / X_{1} \subset W^{*}=\bigvee_{-n}^{n} T^{i}\left(W \vee\left\{F, F^{c}\right\}\right)$. Extend $P$ to $X_{1}^{c}$ so that $P \subset W^{*}$. Then Lemma $6 \stackrel{-n}{\text { implies }}$

(24) $\quad d\left(\bigvee_{0}^{u} T^{i} P, \bigvee_{0}^{u} T_{1}^{i} \bar{R}\right)<\delta_{1}$

(25) $\quad\left\{T^{i} w ; w \in \mathscr{W}_{n} / F, \quad 0 \leqq i \leqq n-1\right\} \subset P^{*}=\bigvee_{-n}^{n} T^{i}\left(P \vee\left\{A, A^{c}\right\}\right)$ because $s^{*}(l(w)), w \in \mathscr{W}_{n} / F$ are distinct. 
We will now check the conclusions (8.4-7). By (1) and (24)

$$
d\left(\bigvee_{0}^{u} T_{1}^{i} R,{\underset{0}{\vee}}_{0}^{u} T^{i} P\right)<\delta .
$$

The choice of $\delta_{1}$ and (24) imply

$$
\left|H\left(\bigvee_{0}^{j} T_{1}^{i} \bar{R}\right)-H\left(\stackrel{\vee}{\vee}_{0}^{i} T^{i} P\right)\right|<\delta / 2, \quad 0 \leqq j \leqq u
$$

which implies (8.6) by (2). Because of (25) and (23), there exists a partition $W^{\prime} C P^{*}$ such that

$$
D\left(W, W^{\prime}\right)=D\left(W, W^{\prime} \mid X_{1}^{c}\right) m\left(X_{1}^{c}\right)<6 \gamma / 10
$$

which implies

$$
h\left(W^{\prime}, T\right)>h(W, T)-\delta / 4
$$

by the choice of $\gamma$. Hence using (10), (23), (6) and (3) in turn

$$
\begin{aligned}
h(P, T) & \geqq h\left(P \vee\left\{A, A^{c}\right\}, T\right)-H\left(\left\{A, A^{c}\right\}\right) \\
& >h\left(P^{*}, T\right)-\delta / 4 \\
& \geqq h\left(W^{\prime}, T\right)-\delta / 4 \\
& >h(W, T)-\delta / 2 \\
& >h\left(\bar{R}, T_{1}\right)-3 \delta / 4>h\left(T_{1}\right)-\delta .
\end{aligned}
$$

On the other hand (10) and (6) imply

$$
\begin{aligned}
h(P, T) & \leqq h\left(W^{*}, T\right) \\
& \leqq h(W, T)+H\left(\left\{F, F^{c}\right\}\right) \\
& <h(W, T)+\beta=h\left(\bar{R}, T_{1}\right) \leqq h\left(T_{1}\right) .
\end{aligned}
$$

Thus (8.5) holds. Next, let $X_{2}=\bigcup_{0}^{n-1} T^{i}(F \cap \cup \mathscr{C})$; hence $m\left(X_{2}^{c}\right)<\varepsilon+r / 10$. Since $p_{j} \cap X_{2}=\cup\left\{T^{i} w ; w \in \mathscr{C} / F, s_{i}^{*}(l(w))=j\right\} \quad$ and $\quad \bar{p}_{j} \cap X_{2}=\cup\left\{T^{i} w\right.$; $\left.w \in \mathscr{C} / F, \bar{s}_{i}(w)=j\right\},(17)$ implies

$$
D\left(P, \bar{P} \mid X_{2}\right) m\left(X_{2}\right)=\sum_{j=1}^{k} m\left(\left(p_{j} \cap X_{2}\right) \triangle\left(\bar{p}_{j} \cap X_{2}\right)\right)
$$




$$
\begin{aligned}
& =2 \sum_{w \in \mathscr{C} \cap F} m(w) \rho\left(s^{*}(l(w)), \bar{s}(w)\right) \\
& \leqq 2 n \varepsilon m(F)<2 \varepsilon .
\end{aligned}
$$

Therefore

$$
\begin{aligned}
D(P, \bar{P}) & =D\left(P, \bar{P} \mid X_{2}\right) m\left(X_{2}\right)+D\left(P, \bar{P} \mid X_{2}^{c}\right) m\left(X_{2}^{c}\right) \\
& <2 \varepsilon+2 \varepsilon+\gamma / 5<5 \varepsilon
\end{aligned}
$$

Thus (5) implies (8.7).

Finally, although we have assumed $h\left(T_{1}\right)>h\left(P^{\prime}, T\right)$ at the beginning of this proof, we can deduce to this case even if $h\left(T_{1}\right)=h\left(P^{\prime}, T\right)$. Indeed, in this case consider a product transformation $S_{1}=T_{1} \times T^{\prime}$ on the product space $X \times Y$ where $T^{\prime}$ is a Bernoulli shift with generator $R^{\prime}=$ $\left\{r_{1}^{\prime}, r_{2}^{\prime}\right\}$ on $Y$. Consider partitions $\widetilde{R}=R \times R^{\prime}$ on $X \times Y$, i.e. $\widetilde{R}=\left(R \times \nu_{Y}\right)$ $\vee\left(\nu_{X} \times R^{\prime}\right)$, and $\hat{P}=P^{\prime} \vee \nu_{X}$ and $\hat{R}=R \vee \nu_{X}$ on $X$, where $\nu_{X}=\{X\}$ and $\nu_{Y}=\{Y\}$ are trivial partitions. Then by choosing the measure of $r_{2}^{\prime}$ so small, we have

$$
\begin{aligned}
& \text { (a) } d\left(\bigvee_{0}^{v} T_{1}^{i} \hat{R}, \bigvee_{0}^{v} S_{1}^{i} \tilde{R}\right)<\eta^{\prime}, \\
& \text { (b) } h\left(T^{\prime}\right)<\eta^{\prime}, \\
& \text { (c) }\left|H\left(\bigvee_{0}^{j} T_{1}^{i} \hat{R}\right)-H\left(\bigvee_{0}^{j} S_{1}^{i} \tilde{R}\right)\right|<\eta^{\prime}, \quad 0 \leqq j \leqq v,
\end{aligned}
$$

where $v=\max \left(u, n_{1}\right)$ and $\eta^{\prime}<\min (\delta / 2, \eta)$. By choosing $\eta^{\prime}$ so small, we have

$$
\begin{aligned}
& d\left(\bigvee_{0}^{n_{1}} S_{1}^{i} \tilde{R},{\underset{0}{\bigvee}}^{n_{1}} T^{i} \hat{P}\right)<\eta, \\
& 0<h\left(S_{1}\right)-h(\hat{P}, T)=h\left(T^{\prime}\right)<\eta, \\
& \left|H\left(\bigvee_{0}^{j} S_{1}^{i} \tilde{R}\right)-H\left(\bigvee_{0}^{j} T^{i} \hat{P}\right)\right|<\eta, \quad 0 \leqq j \leqq n_{1},
\end{aligned}
$$

and $h\left(S_{1}\right)=h\left(\tilde{R}, S_{1}\right)=h\left(T_{1}\right)+h\left(T^{\prime}\right)=h(T)+h\left(T^{\prime}\right)$.

Now we must remark the following three facts. Firstly the proof of this lemma in the case $0<h\left(T_{1}\right)-h\left(P^{\prime}, T\right.$ ) (and $h\left(T_{1}\right)=h(T)$ ) is also 
available even if the space on which $T_{1}$ acts is different from the space of $T$. Secondly the conditions $h\left(T_{1}\right)=h(T)$ and $0<h\left(T_{1}\right)-h\left(P^{\prime}, T\right)$ are only used in taking a partition $W$ satisfying (6). Lastly $n_{1}, n_{2}$, and $\eta$ which are defined in Lemma 5 for $R, T_{1}$ and $\varepsilon^{2} / 3$ remain unaltered even if we replace these by $\tilde{R}, S_{1}$ and $\varepsilon^{2} / 3$. Noting these facts, we can apply the previous argument to $\tilde{R}, S_{1}, n_{1}, n_{2}, \eta, T$ and $\hat{P}$, because we can take a partition $W$ which satisfies a relation similar to (6) by choosing $\eta^{\prime}$ small enough even when $h\left(S_{1}\right)>h(T)=h\left(P^{\prime}, T\right)$. Hence for every $\delta / 2$ we have a finite partition $\tilde{P}$ such that

$$
\begin{gathered}
d\left(\bigvee_{0}^{u} S_{1}^{i} \tilde{R},{\underset{0}{\bigvee}}^{u} T^{i} \tilde{P}\right)<\delta / 2, \\
0<h\left(S_{1}\right)-h(\tilde{P}, T)<\delta / 2, \\
\left|H\left(\bigvee_{0}^{j} S_{1}^{i} \tilde{R}\right)-H\left(\bigvee_{0}^{j} T^{i} \tilde{P}\right)\right|<\delta / 2, \quad 0 \leqq j \leqq u, \\
D(\hat{P}, \tilde{P})<6 \varepsilon .
\end{gathered}
$$

By changing the order of $\tilde{P}$ we have a partition $P$ with the same atoms as $\tilde{P}$ such that $(8.4-7)$ are valid.

Remark. In Lemma 8, without assuming (8.1-3) there exists a partition $P$ which satisfies only (8.4-6).

In the following, we denote $(P, T) \sim\left(R, T_{1}\right)$ if $d\left({\underset{0}{V}}_{0}^{u} T_{1}^{i} R\right)=d\left(\stackrel{u}{V}_{0} T^{i} P\right)$ for all $u \geqq 0$.

Lemma 9. Let $R$ be weak Bernoulli for $T_{1}$ with $h\left(R, T_{1}\right)=h\left(T_{1}\right)$ and $H(R)<\infty$. Given $\varepsilon>0$, there exist $\delta>0$ and a positive integer $u$ such that if $T$ is ergodic with $h(T)=h\left(T_{1}\right)$ and $P^{\prime}$ satisfies

$$
\begin{gathered}
d\left(\bigvee_{0}^{u} T_{1}^{i} R, \stackrel{\vee}{V}_{0}^{u} T^{i} P^{\prime}\right)<\delta, \\
h\left(T_{1}\right)-h\left(P^{\prime}, T\right)<\delta, \\
\left|H\left(\bigvee_{0}^{j} T_{1}^{i} R\right)-H\left(\stackrel{\bigvee}{0}^{j} T^{i} P^{\prime}\right)\right|<\delta, \quad 0 \leqq j \leqq u,
\end{gathered}
$$

then there exists $P$ such that 


$$
\begin{gathered}
(P, T) \sim\left(R, T_{1}\right), \\
D\left(P^{\prime}, P\right)<\varepsilon
\end{gathered}
$$

Proof. Let $n_{1}(\varepsilon)$ and $\eta(\varepsilon)$ be as in Lemma 5 for $R, T_{1}$ and $\varepsilon^{2} / 3$. Let $\eta^{\prime}(\varepsilon)=\min (\eta(\varepsilon), \varepsilon)$ and $\varepsilon^{\prime}=\varepsilon / 12$. Let $\delta=\eta^{\prime}\left(\varepsilon^{\prime}\right)$ and $u=n_{1}\left(\varepsilon^{\prime}\right)$. Applying Lemma 8 for $\eta^{\prime}\left(\varepsilon^{\prime} / 2\right)$ and $u_{1}>\max \left(u, n_{1}\left(\varepsilon^{\prime} / 2\right)\right)$ we get $P_{1}$ such that

$$
\begin{gathered}
d\left(\bigvee_{0}^{⿱_{1}} T_{1}^{i} R, \bigvee_{0}^{u_{1}} T^{i} P_{1}\right)<\eta^{\prime}\left(\varepsilon^{\prime} / 2\right), \\
h\left(T_{1}\right)-h\left(P_{1}, T\right)<\eta^{\prime}\left(\varepsilon^{\prime} / 2\right), \\
\left|H\left(\bigvee_{0}^{j} T_{1}^{i} R\right)-H\left(\bigvee_{0}^{j} T^{i} P_{1}\right)\right|<\eta^{\prime}\left(\varepsilon^{\prime} / 2\right), \quad 0 \leqq j \leqq u_{1}, \\
D\left(P^{\prime}, P_{1}\right)<6 \varepsilon^{\prime} .
\end{gathered}
$$

Assume we have $P_{k}, 1 \leqq k \leqq n$, such that

(1) $\quad d\left(\bigvee_{0}^{u_{k}} T_{1}^{i} R, \bigvee_{0}^{u_{k}} T^{i} P_{k}\right)<\eta^{\prime}\left(\varepsilon^{\prime} / 2^{k}\right)$

(2) $h\left(T_{1}\right)-h\left(P_{k}, T\right)<\eta^{\prime}\left(\varepsilon^{\prime} / 2^{k}\right)$,

(3) $\left|H\left(\bigvee_{0}^{j} T_{1}^{i} R\right)-H\left(\bigvee_{0}^{j} T^{i} P_{k}\right)\right|<\eta^{\prime}\left(\varepsilon^{\prime} / 2^{k}\right), \quad 0 \leqq j \leqq u_{k}$,

(4) $D\left(P_{k-1}, P_{k}\right)<6 \varepsilon^{\prime} / 2^{k-1}$,

where $u_{k}>\max \left(u_{k-1}, n_{1}\left(\varepsilon^{\prime} / 2^{k}\right), k\right)$.

Applying Lemma 8 for $\eta^{\prime}\left(\varepsilon^{\prime} / 2^{n+1}\right)$ and $u_{n+1}>\max \left(u_{n}, n_{1}\left(\varepsilon^{\prime} / 2^{n+1}\right), n+1\right)$ we get $P_{n+1}$ which satisfies (1-4) for $k=n+1$. Thus we have a sequence of partitions $P_{k}, k=1,2, \ldots$, each of which satisfies (1-4). Since the space $(\mathscr{P}, D)$ is complete, (4) implies there exists a partition $P$ such that

$$
D\left(P_{k}, P\right) \rightarrow 0 \text { as } k \rightarrow \infty \text {. }
$$

For each pair $k, n$ such that $u_{k} \geqq n$, (1) implies

$$
d\left(\bigvee_{0}^{n} T_{1}^{i} R,{\underset{V}{V}}_{0}^{n} T^{i} P_{k}\right)<\eta^{\prime}\left(\varepsilon^{\prime} / 2^{k}\right)
$$


Letting $k \rightarrow \infty$ we obtain (9.4). By (4)

$$
D\left(P^{\prime}, P\right) \leqq \sum_{k=1}^{\infty} D\left(P_{k}, P_{k+1}\right)+D\left(P^{\prime}, P_{1}\right)<12 \varepsilon^{\prime}=\varepsilon
$$

Remark. In Lemma 9, without assuming (9.1-3) we have a partition $P$ which satisfies only (9.4).

Thus we have the following

Extended Sinai's Theorem. Let $R$ be a weak Bernoulli generator for $T_{1}$ with $H(R)<\infty$ and $T$ be ergodic with $h\left(T_{1}\right) \leqq h(T)$. Then there exists a partition $P$ such that

$$
(P, T) \sim\left(R, T_{1}\right)
$$

Proof. Choose a partition $Q$ such that $h(Q, T)=h\left(T_{1}\right)$ and $H(Q)<\infty$. Then we can apply Lemma 9 to $T$ acting on $\bigvee_{-\infty}^{\infty} T^{i} Q$ and $T_{1}$, so obtain a partition $P$ which satisfies the conclusion.

Using Lemma 8 we have

Lemma 10. Let $P$ and $Q$ be weak Bernoulli for $T$ respectively and $H(P)<\infty, H(Q)<\infty$. Assume there exists $r>0$ such that

$$
\begin{gathered}
h(P, T)=h(Q, T), \\
Q \subset \bigvee_{-\infty}^{\infty} T^{i} P, \\
P \subset_{-\infty}^{\gamma} \bigvee^{i} Q .
\end{gathered}
$$

Then given $\varepsilon>0$ and $\varepsilon^{\prime}>0$ there exists a partition $P_{1} \subset \bigvee_{-\infty}^{\infty} T^{i} Q$ and $a$ positive integer $K$ such that

$$
\begin{gathered}
\left(P_{1}, T\right) \sim(P, T), \\
Q \complement_{-K}^{\varepsilon} \bigvee^{K} T^{i} P_{1}, \\
D\left(P, P_{1}\right)<2 \gamma+\varepsilon^{\prime},
\end{gathered}
$$




$$
D(Q, \psi(Q))<\varepsilon
$$

where $\psi$ is the canonical map from $(P, T)$ to $\left(P_{1}, T\right)$.

Proof. Let $0<\varepsilon_{1}<\min \left(\varepsilon / 6, \varepsilon^{\prime}\right)$. By $(10.2)$ there exists $K_{1}$ such that $Q \varepsilon_{1}^{\varepsilon_{1}} \underset{-K_{1}}{K_{1}} T^{i} P$. Lemma 9 implies there exists $\delta>0$ and $u$ such that if $P^{\prime} \subset \bigvee_{-\infty}^{\infty} T^{i} Q$ satisfies
(1) $\quad d\left(\stackrel{u}{\vee}_{0}^{i} T^{i} P, \underset{0}{\vee} T^{i} P^{\prime}\right)<\delta$
(2) $h(P, T)-h\left(P^{\prime}, T\right)<\delta$,
(3) $\left|H\left(\bigvee_{0}^{j} T^{i} P\right)-H\left(\stackrel{\bigvee}{0}_{0}^{j} T^{i} P^{\prime}\right)\right|<\delta, \quad 0 \leqq j \leqq u$,

then there exists $P_{1} \subset \vee^{\infty} T^{i} Q$ such that

(4) $\left(P_{1}, T\right) \sim(P, T)$,

(5) $D\left(P, P_{1}\right)<\varepsilon_{1 / 2 K_{1}+1}$.

Let $0<\delta_{1}<\delta / 2$. Choose $0<\varepsilon_{2}<\varepsilon_{1}$ so that $D\left(Q, Q^{\prime}\right)<3 \varepsilon_{2}$ implies $h(Q, T)-\delta_{1}<h\left(Q^{\prime}, T\right)$. Choose $K_{2}>\max \left(K_{1}, u\right)$ such that $Q \complement_{-K_{2}}^{\varepsilon_{2}} T^{K_{2}} P$. Define the $k$-section $P(k)=\left\{p_{1}, \cdots, p_{k-1}, \bigcup_{j \geqq k} p_{j}\right\}$ of $P$. Then there exists $k$ such that $\bar{P}=P(k)$ satisfies

(6) $\quad D(P, \bar{P})<\varepsilon_{1}$,

(7) $Q \stackrel{2 \varepsilon_{j}}{\complement} \underset{-K_{j}}{K_{j}} T^{i} \bar{P}, \quad j=1,2$,

(8) $\quad d\left(\bigvee_{0}^{u} T^{i} P,{\underset{0}{\vee}}^{\natural} T^{i} \bar{P}\right)<\delta_{1}$

(9) $\left|H\left(\bigvee_{0}^{j} T^{i} P\right)-H\left(\bigvee_{0}^{j} T^{i} \bar{P}\right)\right|<\delta_{1}, \quad 0 \leqq j \leqq u$.

Choose $0<\delta_{2}<\min \left(\delta_{1}, \varepsilon_{2}\right)$ so that if $P^{\prime}$ is a partition with $k$ atoms and satisfies $\quad d\left(\bigvee_{0}^{u} T^{i} \bar{P}, \underset{0}{\stackrel{u}{\bigvee}} T^{i} P^{\prime}\right)<\delta_{2}$ then $\quad\left|H\left(\bigvee_{0}^{j} T^{i} \bar{P}\right)-H\left(\bigvee_{0}^{j} T^{i} P^{\prime}\right)\right|<\delta_{1}$ $0 \leqq j \leqq u$. 
Choose $n$ so large that $K_{2} / n<\delta_{2} / 10$. Rohlin's theorem says that there exists a set $F \in \vee^{\infty} T^{i} Q$ such that $T^{i} F,-n \leqq i \leqq n$, are disjoint and $X_{1}=\bigcup^{n} T^{i} F$ satisfies ${ }^{-\infty}\left(X_{1}\right)>1-\delta_{2} / 10$. Define the partition $R=\left\{T^{-n} F\right.$, $\left.T^{-n+1} F, \ldots, T^{n} F, X_{1}^{c}\right\}$. By (10.3) we have a partition $\tilde{P} \subset \bigvee^{\infty} T^{i} Q$ such that $D(P, \tilde{P})<\gamma$.

Consider now the partitions $\bigvee_{-n}^{n} T^{i}(P \vee Q) \vee \tilde{P} \vee R\left(\subset \bigvee_{-\infty}^{\infty} T^{i} P\right)$ and $\bigvee_{-n}^{n} T^{i} Q \vee \tilde{P} \bigvee_{-\infty}^{\infty} R\left(\subset \bigvee_{-\infty}^{\infty} T^{i} Q\right)$. Since $\bigvee_{-\infty}^{\infty} T^{i} Q$ has no atom, there exist partitions $\hat{P}_{i} \subset \bigvee_{-\infty}^{\infty} T^{i} Q,-n \leqq i \leqq n$, satisfying

$$
\begin{aligned}
& \text { (10) } \quad d\left(\bigvee_{-n}^{n} T^{i}(P \vee Q) \vee \tilde{P} \vee R\right)=d\left(\bigvee_{-n}^{n}\left(\hat{P}_{i} \vee T^{i} Q\right) \vee \tilde{P} \vee R\right), \\
& T^{i} \hat{P}_{0} / T^{k+i} F=\hat{P}_{i} / T^{k+i} F
\end{aligned}
$$

for $-n \leqq i \leqq n,-n \leqq k+i \leqq n$. Hence putting $X_{2}=\bigcup_{-n+K_{2}}^{n-K_{2}} T^{i} F$ we have

(12) $m\left(X_{2}\right)>1-\delta_{2} / 5$.

Thus we have

$$
\begin{aligned}
d\left(\bigvee_{0}^{u} T^{i} P,{\underset{0}{\vee}}^{u} T^{i} \hat{P}_{0}\right)= & d\left(\stackrel{\sim}{0}_{0}^{u} \hat{P}_{i},{\underset{0}{V}}^{u} T^{i} \hat{P}_{0}\right) \\
& \leqq 2 m\left(X_{2}^{c}\right)<2 \delta_{2} / 5
\end{aligned}
$$

Let $\bar{P}_{i}=\hat{P}_{i}(k)$ be the $k$-section of $\hat{P}_{i},-n \leqq i \leqq n$. Then (13) implies

(14) $\quad d\left(\bigvee_{0}^{u} T^{i} \bar{P}, \bigvee_{0}^{\stackrel{u}{\bigvee}} T^{i} P_{0}\right)<\delta_{2}$

and so the choice of $\delta_{2}$ implies

(15) $\left|H\left(\bigvee_{0}^{j} T^{i} \bar{P}\right)-H\left(\bigvee_{0}^{j} T^{i} \bar{P}_{0}\right)\right|<\delta_{1}, \quad 0 \leqq j \leqq u$

Because of (7) we have a partition $L^{(j)}$ of the set of all sequences $\left(n_{-K_{j}}, n_{-K_{j}+1}, \cdots, n_{K_{j}}\right), 1 \leqq n_{i} \leqq k,-K_{j} \leqq i \leqq K_{j}$, such that $D\left(L_{\vec{P}}^{(j)}, Q\right)<2 \varepsilon_{j}$, $j=1,2$ (see $\S 2)$. Let $L_{\vec{P}}^{(j)}$ and $L_{\bar{P}_{0}}^{(j)}(j=1,2)$ be defined as in $\S 2$ by $\bigvee_{-K_{j}}^{K_{j}} \bar{P}_{i}$ 
and $\underset{-K_{j}}{K_{j}} T^{i} \bar{P}_{0}$ respectively and $L^{(j)}$. Then (10) implies $D\left(L_{\vec{P}}^{(j)}, Q\right)<2 \varepsilon_{j}$, $j=1,2$, and (11) implies $L_{P_{0}}^{(j)} / X_{2}=L_{\tilde{P}}^{(j)} / X_{2}, j=1,2$. Thus (12) implies

(16) $\quad D\left(L_{\bar{P}_{0}}^{(j)}, Q\right)<2 \varepsilon_{j}+2 m\left(X_{2}^{c}\right)<3 \varepsilon_{j}, \quad j=1,2$.

In particular $D\left(L_{\bar{P}_{0}}^{(2)}, Q\right)<3 \varepsilon_{2}$ which implies

(17) $h(Q, T)-\delta_{1}<h\left(L_{\bar{P}_{0}}^{(2)}, T\right) \leqq h\left(\bar{P}_{0}, T\right)$.

Put $P^{\prime}=\bar{P}_{0}$, then $P^{\prime}$ satisfies (1-3). Indeed (8) and (14) imply (1); (17) and (10.1) imply (2); (9) and (15) imply (3). Hence Lemma 9 implies there exists a partition $P_{1} \subset \vee^{\infty} T^{i} Q$ satisfying (4) and (5). Let $P^{*}=P_{1}(k)$ the $k$-section of $P_{1}$ and let $L_{P^{*}}^{(1)}$ be defined in the way of $\S 2$ by $\underset{-K_{1}}{K_{1}} T^{i} P^{*}$ and $L^{(1)}$. Then by (5)

(18) $\quad D\left(L_{P^{*}}^{(1)}, L_{\bar{P}_{0}}^{(1)}\right) \leqq D\left(\bigvee_{-K_{1}}^{K_{1}} T^{i} P^{*}, \bigvee_{-K_{1}}^{K_{1}} T^{i} \bar{P}_{0}\right)$

$$
\leqq\left(2 K_{1}+1\right) D\left(P^{*}, \bar{P}_{0}\right)<\varepsilon_{1}
$$

and so (16) implies

$$
D\left(Q, L_{P^{*}}^{(1)}\right)<4 \varepsilon_{1}<\varepsilon
$$

which implies (10.5) for $K=K_{1}$. Since (10) and (6) we have

$$
\begin{aligned}
D\left(P, P_{1}\right) & \leqq D(P, \tilde{P})+D\left(\tilde{P}, \hat{P}_{0}\right)+D\left(\hat{P}_{0}, \bar{P}_{0}\right) \\
& =2 D(P, \tilde{P})+D(P, \bar{P})<2 \gamma+\varepsilon_{1}
\end{aligned}
$$

which implies (10.6). Finally note $\phi\left(L_{\bar{P}}^{(1)}\right)=L_{P^{*}}^{(1)}$. Then (16), (18) and the choice of $L^{(1)}$ imply

$$
\begin{aligned}
D(Q, \psi(Q)) & \leqq D\left(Q, L_{\bar{P}_{0}}^{(1)}\right)+D\left(L_{\bar{P}_{0}}^{(1)}, L_{P^{*}}^{(1)}\right)+D\left(\psi\left(L_{\bar{P}}^{(1)}\right), \psi(Q)\right) \\
& <3 \varepsilon_{1}+\varepsilon_{1}+2 \varepsilon_{1}<\varepsilon .
\end{aligned}
$$

Lemma 11. Let $R$ and $P$ be weak Bernoulli generators for $T_{1}$ and $T$ respectively and $h\left(T_{1}\right)=h(T)$ and $H(R)<\infty, H(P)<\infty$. Let $Q$ be a 
partition such that

$$
(Q, T) \sim\left(R, T_{1}\right)
$$

Given $\varepsilon>0$, there exist a partition $Q_{1}$ and a positive integer $K$ such that

$$
\begin{aligned}
& \left(Q_{1}, T\right) \sim\left(R, T_{1}\right), \\
& P \bigodot_{-K}^{\varepsilon} \bigvee^{K} T^{i} Q_{1}, \\
& D\left(Q, Q_{1}\right)<\varepsilon .
\end{aligned}
$$

Proof. Let $0<\varepsilon_{1}<\varepsilon / 4$. Because of (11.1) we can apply Lemma 10 to obtain $P_{1} \subset \bigvee_{-\infty}^{\infty} T^{i} Q$ such that

(1) $\left(P_{1}, T\right) \sim(P, T)$,

(2) $Q \complement_{-\infty}^{\varepsilon_{1}} \bigvee_{-\infty}^{\infty} T^{i} P_{1}$

(3) $D(Q, \psi(Q))<\varepsilon_{1}$,

where $\psi$ is the canonical map from $(P, T)$ to $\left(P_{1}, T\right)$. Now (1) implies $h(Q, T)=h(P, T)=h\left(P_{1}, T\right)$. We can again apply Lemma 10 to obtain $Q^{\prime} \subset \bigvee_{-\infty}^{\infty} T^{i} P_{1}$ and $K>0$ such that
(4) $\left(Q^{\prime}, T\right) \sim(Q, T) \sim\left(R, T_{1}\right)$,
(5) $P_{1} \complement_{-K}^{\varepsilon_{1}} \bigvee_{-K}^{K} T^{i} Q^{\prime}$,
(6) $D\left(Q, Q^{\prime}\right)<3 \varepsilon_{1}$.

Let $Q_{1}=\psi^{-1}\left(Q^{\prime}\right)$. Then (4) implies (11.2), (5) implies (11.3), and (3) and (6) imply

$$
D\left(Q, Q_{1}\right)=D\left(\psi(Q), Q^{\prime}\right) \leqq D(\psi(Q), Q)+D\left(Q, Q^{\prime}\right)<4 \varepsilon_{1}<\varepsilon .
$$

Now we can prove Proposition 1.

Proof of Proposition 1. Let $R$ and $P$ be weak Bernoulli generators 
for $T_{1}$ and $T$ respectively with $h\left(T_{1}\right)=h(T)$ and $H(R)<\infty, H(P)<\infty$. By the Extended Sinai's Theorem there exists a partition $Q$ such that $(Q, T) \sim\left(R, T_{1}\right)$. Then Lemma 11 implies that for $\varepsilon_{1}<2^{-1}$ we can find a partition $Q_{1}$ and $K_{1}$ such that

(1) $\left(Q_{1}, T\right) \sim\left(R, T_{1}\right)$,

(2) $D\left(Q, Q_{1}\right)<\varepsilon_{1}$,

(3) $P \mathrm{2}^{-1} \bigvee_{-K_{1}}^{K_{1}} T^{i} Q_{1}$

Choose $\varepsilon_{2}<2^{-2}$ so small that (3) and

(4) $D\left(Q_{1}, Q_{2}\right)<\varepsilon_{2}$

imply

(5) $P \stackrel{2^{-1}+2^{-2}}{\complement} \bigvee_{-K_{1}}^{K_{1}} T^{i} Q_{2}$.

Apply Lemma 11 to obtain $Q_{2}$ and $K_{2}$ satisfying (4),

(6) $\left(Q_{2}, T\right) \sim\left(R, T_{1}\right)$,

(7) $P \stackrel{2^{-2}}{\complement} \underset{-K_{2}}{K_{2}} T^{i} Q_{2}$.

Suppose now we have got $Q_{n}$ and $K_{1}, \ldots, K_{n}$ such that

(8) $\left(Q_{n}, T\right) \sim\left(R, T_{1}\right)$,

(9) $\quad P \stackrel{2^{-i}+\cdots+2^{-n}}{\complement} \bigvee_{-K_{j}}^{K_{j}} T^{i} Q_{n}, \quad 1 \leqq j \leqq n$.

Choose $\varepsilon_{n+1}<2^{-n-1}$ so small that (9) and

(10) $D\left(Q_{n}, Q_{n+1}\right)<\varepsilon_{n+1}$

imply

(11) $P^{2^{-j}+\cdots+2^{-n-1}} \bigvee_{-K_{j}}^{K_{j}} T^{i} Q_{n+1}, \quad 1 \leqq j \leqq n$.

Apply Lemma 11 to obtain $Q_{n+1}$ and $K_{n+1}$ satisfying (10), 
(12) $\left(Q_{n+1}, T\right) \sim\left(R, T_{1}\right)$

(13) $P \stackrel{2^{-n-1}}{\complement} \bigvee_{-K_{n+1}}^{K_{n+1}} T^{i} Q_{n+1}$.

Thus by induction we have the sequence of partitions $Q_{n}, n=1,2, \ldots$, which satisfy (8), (9) and (10). Then (10) implies there exists a countable partition $Q_{\infty}$ such that

$$
D\left(Q_{n}, Q_{\infty}\right) \rightarrow 0 \text { as } n \rightarrow \infty
$$

Then (8) implies

(14) $\left(Q_{\propto}, T\right) \sim\left(R, T_{1}\right)$,

and (9) implies

(15) $\quad P \subset \bigvee_{-\infty}^{\infty} T^{i} Q_{\infty}$

Since $P$ is a generator, the proposition follows from (14) and (15).

\section{§4. General Case}

In this section, using the results of the preceding section, we will prove the following theorem of Ornstein.

Theorem. Two generalized weak Bernoulli transformations with the same entropy (including the case $\infty$ ) are isomorphic.

To prove this theorem, we must prepare some lemmas. The essential one is Lemma 14 which is similar to Lemma $4^{\prime}$ in [5] and is proved by some modified argument. Using this lemma and Lemma 11 we can get Lemma 15 and 16 respectively, which prove our theorem. For completeness we will state the proofs of these lemmas.

Lemma 12. Let $\varepsilon>0$ and $\zeta=\zeta(\varepsilon)$ as in Lemma 2. If countable partitions $P$ and $R$ satisfy

$$
H(P)+H(R)-h(P \vee R, T)<\zeta,
$$


then

$$
T^{i} P \stackrel{\varepsilon}{\perp} \bigvee_{0}^{n} T^{j} R \vee \stackrel{i-1}{\bigvee}_{0}^{j} P, \quad 0 \leqq i \leqq n
$$

Remark. This conclusion implies $P \stackrel{\varepsilon}{\perp} \bigvee_{0}^{n} T^{j} R$ when $i=0$.

Proof. Noting the following relations

$$
\begin{aligned}
& h(P \vee R, T)=\lim _{n \rightarrow \infty} \frac{1}{n+1}\left[H\left(\bigvee_{0}^{n} T^{j} R\right)+\sum_{0}^{n} H\left(T^{i} P \mid \bigvee_{0}^{n} T^{j} R \vee \bigvee_{0}^{i-1} T^{j} P\right)\right], \\
& h(R, T)=\lim _{n \rightarrow \infty} \frac{1}{n+1} H\left(\bigvee_{0}^{n} T^{j} R\right), \\
& H\left(T^{k} P \mid \bigvee_{0}^{m+n} T^{j} R \vee \bigvee_{0}^{i-1} T^{j} P\right) \leqq H\left(T^{i} P \mid \bigvee_{0}^{n} T^{j} R \vee \bigvee_{0}^{i-1} T^{j} P\right),
\end{aligned}
$$

for $m>0, i \leqq k \leqq i+m$, we have

$$
\begin{aligned}
h(P \vee R, T) & \leqq h(R, T)+H\left(T^{i} P \mid{ }_{0}^{n} T^{j} R \vee{ }_{0}^{i-1} T^{j} P\right) \\
& \leqq H(R)+H\left(T^{i} P \mid \stackrel{\vee}{\vee}_{0}^{n} R \vee \bigvee_{0}^{i-1} T^{j} P\right)
\end{aligned}
$$

for any $n \geqq 0,0 \leqq i \leqq n$. Then the assumption and the above inequality imply

$$
H(P)-H\left(T^{i} P \mid \bigvee_{0}^{n} T^{j} R \vee \bigvee_{0}^{i-1} T^{j} P\right)<\zeta, \quad 0 \leqq i \leqq n
$$

Therefore the lemma follows from Lemma 2.

Lemma 13. Let $\varepsilon>0$ and $\varepsilon^{\prime}>0$. Let $\left\{P_{i}\right\},\left\{R_{i}\right\}, 0 \leqq i \leqq n$, be the sequences of countable partitions and $\pi$ be a probability vector such that

$$
\begin{gathered}
P_{i} \perp{ }_{0}^{\varepsilon} \mathbb{V}_{j} \bigvee \bigvee_{0}^{i-1} P_{j}, \quad 0 \leqq i \leqq n \\
\left\{R_{i}\right\}, \quad 0 \leqq i \leqq n, \text { are independent } \\
d\left(P_{i}, \pi\right)<\varepsilon^{\prime}, \quad 0 \leqq i \leqq n
\end{gathered}
$$


Then there exists a sequence of partitions $\left\{\bar{P}_{i}\right\}, 0 \leqq i \leqq n$, satisfying

$$
\begin{gathered}
\left\{\bar{P}_{i}, R_{j}\right\}, 0 \leqq i, j \leqq n, \text { are independent } \\
d\left(\bar{P}_{i}\right)=\pi, \quad 0 \leqq i \leqq n \\
D\left(P_{\imath}, \bar{P}_{i}\right)<\varepsilon+\varepsilon^{\prime}, \quad 0 \leqq i \leqq n
\end{gathered}
$$

Proof. Note that the following statement is useful for the proof; if $P \perp Q$ and $d(P, \pi)<\varepsilon^{\prime}$, then there exists a partition $\bar{P}$ such that $\bar{P}$ and $Q$ are independent, $d(\bar{P})=\pi$, and $D(P, \bar{P})<\varepsilon+\varepsilon^{\prime}$. Using this fact we can easily prove this lemma by induction.

Lemma 14. Let $T$ be an ergodic transformation and $h(T)<\infty$. Let $\varepsilon>0, \zeta=\zeta(\varepsilon)$ be as in Lemma 2, and $\theta(\varepsilon)=\min \left(\zeta\left(\varepsilon^{2} / 2\right), \varepsilon^{2} / 2\right) / 2$. If a probability vector $\pi=\left\{\pi_{i} ; i \geqq 1\right\}$ and countable partitions $P$ and $R$ satisfy

$$
\begin{gathered}
\left\{T^{i} R\right\},-\infty<i<\infty, \text { are independent } \\
H(\pi)=h(T)-H(R), \\
d(P, \pi)<\theta(\varepsilon), \\
H(P)+H(R)-h(P \vee R, T)<\theta(\varepsilon),
\end{gathered}
$$

then given $\delta>0$ there exists a (finite) partition $\tilde{P}$ such that

$$
\begin{gathered}
d(\tilde{P}, \pi)<\delta, \\
H(\tilde{P})+H(R)-h(\tilde{P} \vee R, T)<\delta, \\
D(P, \tilde{P})<15 \varepsilon .
\end{gathered}
$$

Proof. We may assume $\delta<\theta(\varepsilon)$. Let $\pi^{\prime}=\left\{\pi_{i}^{\prime} ; 1 \leqq i \leqq l\right\}$ be a (finite) probability vector such that the following two conditions are satisfied;

(1) $d\left(\pi, \pi^{\prime}\right)=\sum_{i}\left|\pi_{i}-\pi_{i}^{\prime}\right|<\frac{\delta}{4}$, 
(2) $H\left(\pi^{\prime}\right)=H(\pi)+\alpha, \quad 0<\alpha<\frac{\delta}{4}$.

By the assumption (14.4) we can apply Lemma 12 and we get, for every $n \geq 0$,

(3) $\quad T^{i} P \stackrel{\varepsilon^{2} / 2}{\perp} \bigvee_{0}^{n} T^{j} R \vee \stackrel{i-1}{\bigvee}_{0}^{j} P, \quad 0 \leqq i \leqq n$

and using (14.3) and (1), we get

(4) $\quad d\left(T^{i} P, \pi^{\prime}\right)<\varepsilon^{2} / 2$.

So by (3), (14.1) and (4) we can apply Lemma 13 to get a sequence of partitions $\left\{\bar{P}_{i} ; 0 \leqq i \leqq n\right\}$ such that

(5) $\left\{\bar{P}_{i}, T^{j} R ; 0 \leqq i, j \leqq n\right\} \quad$ are independent,

(6) $\quad d\left(\bar{P}_{i}\right)=\pi^{\prime}, \quad 0 \leqq i \leqq n$,

(7) $\quad D\left(\bar{P}_{i}, T^{i} P\right)<\varepsilon^{2}, \quad 0 \leqq i \leqq n$.

By the ergodicity, $T$ has a countable generator, so that we have a refinement $Q$ of $P \vee R$ satisfying

(8) $h(Q, T)=h(T)$.

Choose $0<\varepsilon^{\prime}<\min (1 / 2, \varepsilon / 2, \delta / 12)$ so small that the following two conditions are valid;

(9) $D\left(Q, Q^{\prime}\right)<10 \varepsilon^{\prime}$ implies $h(Q, T)-\delta / 4<h\left(Q^{\prime}, T\right)$,

(10) $\quad d\left(\tilde{P}, \pi^{\prime}\right)<10 \varepsilon^{\prime}$ and $N(\tilde{P}) \leqq l$ imply

$$
\left|H(\tilde{P})-H\left(\pi^{\prime}\right)\right|<\delta / 4 \text {. }
$$

Choose $n>3 / \alpha$ so large that the following three statements are valid: (A) By Shannon-McMillan-Breiman theorem setting $\bar{Q}=\bigvee_{0}^{n-1} T^{k} Q$ there exists a $\bar{Q}$-measurable set $Y$ such that

(11) $m(Y)>1-\varepsilon^{\prime}$, 
(12)

$$
2^{-n(h(T)+\alpha / 3)}<m(B)<2^{-n(h(T)-\alpha / 3)}, \quad B \in \bar{Q} / Y,
$$

(B) we will denote the $\bar{P} \vee R$-name of $x \in X$ as $\bar{s}(x)=\left\{\left(\bar{s}_{k}(x), t_{k}(x)\right)\right.$; $0 \leqq k \leqq n-1\}$ when $x \in \bigcap_{0}^{n-1} \bar{p}_{s_{k}}^{k}(x) \cap T^{k} r_{t_{k}}(x)$ for $\bar{P}_{k}=\left\{\bar{p}_{i}^{k} ; 1 \leqq i \leqq l\right\}$ and $R=\left\{r_{i} ; i \geqq 1\right\}$. Let $N(j ; \bar{P}, x)$ be the number of $k$ which satisfies $\bar{s}_{k}(x)=j$. Then $N(j ; \bar{P}, G)$ is defined naturally for $G \in \bigvee_{0}^{n-1}\left(\bar{P}_{k} \bigvee T_{n-1}^{k} R\right)$. Using the law of large numbers, by (5) and (6), setting ${ }^{0} \hat{P}=\bigvee_{0}^{n-1}\left(\bar{P}_{k} \vee T^{k} R\right)$, there exists a $\hat{P}$-measurable set $\bar{Y}$ such that

(13) $m(\bar{Y})>1-\varepsilon^{\prime}$

(14) $2^{-n\left(H\left(\pi^{\prime}\right)+H(R)+\alpha / 3\right)}<m(G)<2^{-n\left(H\left(\pi^{\prime}\right)+H(R)-\alpha / 3\right)}, \quad G \in \hat{P} / \bar{Y}$,

(15) $\left|N(j ; \bar{P}, G) / n-\pi_{j}^{\prime}\right|<\varepsilon^{\prime} / l, \quad G \in \hat{P} / \bar{Y}$,

(C) $m(A)<1 / n$ implies $H\left(\left\{A, A^{c}\right\}\right)<\delta / 4$. By the choice of $n$ in (A) and $(B)$, we can estimate

(16) $N(\bar{Q} / Y)<2^{n(h(T)+\alpha / 3)}$,

(17) $N(\hat{P} / \bar{Y})>2^{n(h(T)+\alpha / 3)}$.

Now we will also define the $P \vee R$-name $s(x)=\left\{\left(s_{k}(x), t_{k}(x)\right) ; 0 \leqq\right.$ $k \leqq n-1\}$ of $x \in X$ by the same way as above. Note that this name is the same in every atom of $\bigvee_{0}^{n-1} T^{k} Q$. Let $E_{k}$ denote the set of all points $x$ such that $\bar{s}_{k}(x) \neq s_{k}(x)$ and $D_{e}$ denote the set of all points $x$ for which $\bar{s}_{k}(x)$ ard $s_{k}(x)$ are distinct in at least $e$ places, then we have $\sum_{0}^{n-1} m\left(E_{k}\right) \geqq$ $e m\left(D_{e}\right)$. On the other hand, for $P=\left\{p_{i} ; i \geqq 1\right\}$ and $\bar{P}_{k}=\left\{\bar{p}_{i}^{k} ; 1 \leqq i \leqq l\right\}$, we have $D\left(T^{k}(P \vee R), \bar{P}_{k} \vee T^{k} R\right)=2 m\left(E_{k}\right)$. Then setting $W=D_{n \varepsilon}^{c}$ we have

(18) $m(W)>1-\varepsilon$.

Let $\mathscr{B}$ be the collection of atoms $B$ in $\bar{Q} / Y$ which satisfy $m(B \cap W \cap \bar{Y}) \geqq$ $m(B) / 2$ and let $Z=\cup \mathscr{B}$ the union of sets in $\mathscr{B}$. Then we can estimate

(19) $m\left(Z^{c}\right)<2\left(\varepsilon+\varepsilon^{\prime}\right)<3 \varepsilon$.

Now we can assign to each atom $B \in \mathscr{B}$ an atom $G=\psi(B) \in \hat{P} / \bar{Y}$ 
such that $B \cap G \cap W \neq \varnothing$ in a $1-1$ manner. This can be done by the same way as in the proof of Lemma 8 using the marriage lemma because of (16) and (17). Note that two names $s(B)$ and $\bar{s}(\psi(B))$ are the same in the second component and are distinct in at most $n \varepsilon$ places in the first component. We can extend $\psi$ to a 1-1 mapping from $\bar{Q} / Y$ into $\hat{P} / \bar{Y}$ by (16) and (17).

Using Rohlin's theorem and the same argument as in the proof of Lemma 8 , we get a measurable set $F$ such that $T^{-k} F, 0 \leqq k \leqq n-1$, are disjoint, $m\left(\bigcup_{0}^{n-1} T^{-k} F\right)>1-\varepsilon^{\prime}$ and

$$
\begin{aligned}
& m\left(\bigcup_{0}^{n-1} T^{-k}(F \cap Z)\right)>1-\varepsilon^{\prime}-6 \varepsilon \\
& m\left(\bigcup_{0}^{n-1} T^{-k}(F \cap Y)\right)>1-3 \varepsilon^{\prime} .
\end{aligned}
$$

We will now define the partition $\tilde{P}=\left\{p_{1}, p_{2}, \cdots, p_{l}\right\}$ as follows. Let $X_{1}=\bigcup_{0}^{n-1} T^{-k}(F \cap Y)$ and $A=F \cap Y$. For simplicity we denote the $\bar{P} \vee R$ -name of $\psi(B), B \in \bar{Q} / Y$ as $\bar{s}(B)=\left\{\left(\bar{s}_{k}(B), t_{k}(B)\right) ; 0 \leqq k \leqq n-1\right\}$. Define $\tilde{P}$ on $T^{-k} A, \quad 0 \leqq k \leqq n-1$, by

$$
\tilde{p}_{j} \cap T^{-k} A=\cup\left\{T^{k}(F \cap B) ; B \in \bar{Q} / Y, s_{k}(B)=j\right\}, \quad 1 \leqq j \leqq l .
$$

And extend $\tilde{P}$ to $X_{1}^{c}$ so that $X_{1}^{c} \subset \tilde{p}_{1}$. Then this partition $\tilde{P}$ satisfies (14.5-7). Indeed

$$
\begin{aligned}
m\left(\tilde{p}_{j}\right) & =\sum_{k=0}^{n-1}\left[m\left(\tilde{p}_{j} \cap T^{-k} A\right)+m\left(\tilde{p}_{j} \cap X_{1}^{c}\right)\right] \\
& =\sum_{k=1}^{n-1}\left[\sum_{\substack{B \in \bar{Q}^{\prime} \mid Y \\
s_{k}(B)=j}} m(F \cap B)+m\left(\tilde{p}_{j} \cap X_{1}^{c}\right)\right] \\
& =\sum_{B \in \bar{Q} \mid Y} N(j: \bar{P}, B) m(F \cap B)+m\left(X_{1}^{c}\right),
\end{aligned}
$$

by (15) and (21) we have

$$
\begin{aligned}
d\left(\tilde{P}, \pi^{\prime}\right) & \leqq \sum_{j=1}^{l}\left|m\left(\tilde{p}_{j}\right)-\pi_{j}^{\prime}\right| \\
& <\sum_{B \in \bar{Q}_{\mid} \mid Y} n m(F \cap B) \sum_{j=1}^{l}\left|N(j ; \bar{P}, B) / n-\pi_{j}^{\prime}\right|+2 m\left(X_{1}^{c}\right)
\end{aligned}
$$




$$
<7 \varepsilon^{\prime}<\frac{3}{4} \delta
$$

so by (1) we have (14.5);

$$
d(\tilde{P}, \pi) \leqq d\left(\tilde{P}, \pi^{\prime}\right)+d\left(\pi^{\prime}, \pi\right)<\delta
$$

Secondly the choice (C) of $n$ implies

$$
H\left(\left\{A, A^{c}\right\}\right)<\delta / 4
$$

On the other hand putting $P^{*}=\bigvee_{-n}^{n} T^{k}\left(\tilde{P} \vee\left\{A, A^{c}\right\} \vee R\right)$ we have

$$
\left\{T^{-k}(F \cap B) ; \quad 0 \leqq k \leqq n-1, B \in \bar{Q} / Y\right\} \subset P^{*},
$$

so we have a subpartition $Q^{\prime}$ of $P^{*}$ which coincides with $Q$ on $X_{1}$ so that $D\left(Q, Q^{\prime}\right) \leqq 2 m\left(X_{1}^{c}\right)<8 \varepsilon^{\prime}$. Therefore by (8) and (9), these relations imply

$$
\begin{aligned}
h(T)=h(Q, T) & <h\left(Q^{\prime}, T\right)+\delta / 4 \\
& \leqq h\left(P^{*}, T\right)+\delta / 4 \\
& \leqq h(\tilde{P} \vee R, T)+H\left(\left\{A, A^{c}\right\}\right)+\delta / 4 \\
& <h(\tilde{P} \vee R, T)+\delta / 2,
\end{aligned}
$$

and (22) implies

$$
\left|H(\tilde{P})-H\left(\pi^{\prime}\right)\right|<\delta / 4
$$

so by (2) we have (14.6);

$$
H(\tilde{P})+H(R)-h(\tilde{P} \vee R, T)<\delta
$$

Finally noting the similarity of the following two relations

$$
\tilde{p}_{j} \cap T^{-k}(B \cap F)=\left\{\begin{array}{cc}
T^{-k}(B \cap F), & \bar{s}_{k}(B)=j \\
\varnothing \quad, & \bar{s}_{k}(B) \neq j,
\end{array}\right.
$$




$$
p_{j} \cap T^{-k}(B \cap F)=\left\{\begin{array}{cc}
T^{-k}(B \cap F), & s_{k}(B)=j \\
\varnothing \quad, & s_{k}(B) \neq j
\end{array}\right.
$$

and noting the estimation by (20)

$$
m\left(X_{2}\right)<\varepsilon^{\prime}+6 \varepsilon \quad \text { where } \quad X_{2}=X \backslash \bigcup_{k=0}^{n-1} T^{-k}(F \cap Z),
$$

we have

$$
\begin{aligned}
D(P, \tilde{P})= & \sum_{j} \sum_{B \in \mathscr{B}} \sum_{k=0}^{n-1} m\left[\left(p_{j} \cap T^{-k}(B \cap F)\right) \triangle\left(\tilde{p}_{j} \cap T^{-k}(B \cap F)\right)\right] \\
& +D\left(P, \tilde{P} \mid X_{2}\right) m\left(X_{2}\right) \\
\leqq & \sum_{B} \sum_{k ; \bar{s}_{k}(B)+s_{k}(B)} 2 m\left(T^{-k}(B \cap F)\right)+13 \varepsilon \\
\leqq & 2 n \varepsilon \sum_{B \in \mathscr{B}} m(B \cap F)+13 \varepsilon<15 \varepsilon .
\end{aligned}
$$

Remark. If we only assume (14.1) and (14.2), we obtain $\tilde{P}$ which satisfies (14.5) and (14.6).

Using this lemma we can prove the following

Lemma 15. Let $T$ be an ergodic transformation and $h(T)<\infty$. If a partition $R$ satisfies

$$
\begin{gathered}
\left\{T^{i} R\right\},-\infty<i<\infty, \text { are independent } \\
H(R) \leqq h(T)
\end{gathered}
$$

then there is a (finite) partition $\tilde{P}$ such that

$$
H(\tilde{P})+H(R)=h(T)
$$

$$
\left\{T^{i}(\tilde{P} \vee R)\right\},-\infty<i<\infty \text {, are independent and } \tilde{P} \text { and } R \text { are }
$$
independent.

Proof. Take a (finite) probability vector $\pi=\left\{\pi_{i} ; 1 \leqq i \leqq l\right\}$ such that $H(\pi)=h(T)-H(R)$. Then, by the previous remark we can apply Lemma 
14 to get a (fiinite) partition $P_{0}$ for every $\delta_{0}$ such that
(1) $d\left(P_{0}, \pi\right)<\theta\left(\delta_{0}\right)$,
(2) $H\left(P_{0}\right)+H(R)-h\left(P_{0} \vee R, T\right)<\theta\left(\delta_{0}\right)$,

where $\theta\left(\delta_{0}\right)$ is defined in Lemma 14 . Choose $\delta_{n} \searrow 0, n \geqq 1$, we can apply Lemma 14 to get a partition $P_{1}$ for $\theta\left(\delta_{1}\right)$ such that
(3) $d\left(P_{1}, \pi\right)<\theta\left(\delta_{1}\right)$,
(4) $H\left(P_{1}\right)+H(R)-h\left(P_{1} \vee R, T\right)<\theta\left(\delta_{1}\right)$,
(5) $D\left(P_{0}, P_{1}\right)<15 \delta_{0}$.

Then we obtain a sequence of partitions $\left\{P_{i}\right\}, i \geqq 0$, by induction such that

(6) $\quad d\left(P_{j}, \pi\right)<\theta\left(\delta_{j}\right), \quad j=0,1, \cdots, n, \ldots$

(7) $H\left(P_{j}\right)+H(R)-h\left(P_{j} \vee R, T\right)<\theta\left(\delta_{j}\right), \quad j=0,1, \ldots, n, \ldots$

(8) $\quad D\left(P_{j-1}, P_{j}\right)<15 \delta_{j-1}, \quad j=1,2, \ldots, n, \ldots$

Choosing $\delta_{n}$ so small that (8) implies the existence of a partition $\tilde{P}$ such that $D\left(P_{n}, \tilde{P}\right) \rightarrow 0$ as $n \rightarrow \infty$. Then $d(\tilde{P})=\pi$ and $\tilde{P}$ is a finite partition and satisfies (15.3). On the other hand for every $n \geqq 0$ we will take $A \in T^{n}(\tilde{P} \bigvee R)$ and $B \in \bigvee_{0}^{n-1} T^{i}(\tilde{P} \vee R)$, and take $A_{k} \in T^{n}\left(P_{k} \bigvee R\right)$ and $B_{k} \in$ $\bigvee_{0}^{n-1} T^{i}\left(P_{k} \vee R\right)$ corresponding to $A$ and $B$ respectively for every $k \geqq 0$. Then

$$
\begin{aligned}
& \left|m(A \cap B)-m\left(A_{k} \cap B_{k}\right)\right| \leqq(n+1) D\left(\tilde{P}, P_{k}\right) \rightarrow 0, \quad k \rightarrow \infty, \\
& \left|m(A)-m\left(A_{k}\right)\right| \rightarrow 0, \quad\left|m(B)-m\left(B_{k}\right)\right| \rightarrow 0, \quad k \rightarrow \infty,
\end{aligned}
$$

and by (7) Lemma 12 says

$$
T^{n} P_{k} \stackrel{\delta_{k / 2}^{2}}{\perp} \underset{0}{\bigvee} T^{j} R \bigvee \bigvee_{0}^{n-1} T^{j} P_{k}, \quad T^{n} R \stackrel{\delta_{k / 2}^{2}}{\perp} \underset{0}{\bigvee} T^{j} P_{k} \bigvee \bigvee_{0}^{n-1} T^{j} R
$$

Therefore we have 


$$
T^{n}\left(P_{k} \bigvee R\right) \stackrel{3 \delta_{k}^{2} / 2}{\perp} \bigvee_{0}^{n-1} T^{j}\left(P_{k} \bigvee R\right)
$$

and then

$$
\left|m\left(A_{k} \cap B_{k}\right)-m\left(A_{k}\right) m\left(B_{k}\right)\right|<\frac{3}{2} \delta_{k}^{2} \rightarrow 0 \text { as } k \rightarrow \infty .
$$

Hence $T^{n}(\tilde{P} \vee R)$ and $\bigvee_{0}^{n-1} T^{i}(\tilde{P} \vee R)$ are independent. Similarly we can prove the independence of $\tilde{P}$ and $R$. Thus we have (15.4).

Remark. If $H(R)=h(T)$, then we take the trivial partition as $\tilde{P}$.

For proving our theorem it is convenient to state a special case of Lemma 11 in the following

Lemma 16. Let $P$ be a weak Bernoulli generator for $T$ and $H(P)$ $<\infty$. Let $Q$ be weak Bernoulli for $T$ such that $h(Q, T)=h(T)$ and $H(Q)<\infty$. Given $\varepsilon>0$, there exist a partition $Q_{1}$ and $a$ positive integer $K$ such that

$$
\begin{gathered}
\left(Q_{1}, T\right) \sim(Q, T) \\
P \stackrel{\varepsilon}{\subset}{\underset{-K}{V}}_{V}^{K} T^{i} Q_{1} \\
D\left(Q, Q_{1}\right)<\varepsilon
\end{gathered}
$$

Proof of the theorem. Let $P$ be a generalized weak Bernoulli generator for $T$. Take an increasing sequence of finite partitions $\left\{P_{n}\right\}, n \geqq 1$, such that each $P_{n}$ is weak Bernoulli for $T$ and $\bigvee_{n=1}^{\infty} P_{n}=P$ (hence $h\left(P_{n}, T\right) \nearrow$ $h(P, T)=h(T))$.

Since $P_{1}$ is weak Bernoulli for $T$ and $H\left(P_{1}\right)<\infty$, there exists a finite partition ${ }_{1} Q_{1}$ by Proposition 1 such that

(1) ${ }_{1} Q_{1} \subset \bigvee_{-\infty}^{\infty} T^{i} P_{1}$

(2) $\left\{T_{1}^{i} Q_{1}\right\},-\infty<i<\infty$, are independent

(3) $\bigvee_{-\infty}^{\infty} T_{1}^{i} Q_{1}=\bigvee_{-\infty}^{\infty} T^{i} P_{1} . \quad\left(\right.$ Hence $H\left({ }_{1} Q_{1}\right)=h\left(P_{1}, T\right)$.) 
Applying Lemma 15 for $P_{2}$ and $T$, we have a finite partition ${ }_{1} Q_{2} C$ $\bigvee_{-\infty}^{\infty} T^{i} P_{2}$ such that

(4) $H\left({ }_{1} Q_{1}\right)+H\left({ }_{1} Q_{2}\right)=h\left(P_{2}, T\right)$

(5) $\left\{T^{i}\left({ }_{1} Q_{1} \vee{ }_{1} Q_{2}\right)\right\},-\infty<i<\infty$, are independent and ${ }_{1} Q_{1}$ and ${ }_{1} Q_{2}$ are independent.

Let $0<\varepsilon_{1}<2^{-1}$ and take $K_{1}>0$ such that

(6) $P_{1} \stackrel{\varepsilon_{1}}{K_{-K_{1}}^{K_{1}}} T_{1}^{i} Q_{1}$

Choosing $0<\varepsilon_{2}<2^{-2}$ so small that the following statement holds.

(7) $D\left({ }_{1} Q_{1},{ }_{2} Q_{1}\right)<\varepsilon_{2}$ implies $P_{1} \stackrel{2}{-1}^{K_{1}} T_{-K_{1}}^{i} Q_{2} Q_{1}$.

Applying Lemma 16 for $P_{2}, \quad T$ and ${ }_{1} Q_{1} \vee_{1} Q_{2}$, we have partitions ${ }_{2} Q_{1},{ }_{2} Q_{2} \subset \bigvee_{-\infty}^{\infty} T^{i} P_{2}$ and $K_{2}>0$ such that

(8) $\left\{T^{i}\left({ }_{2} Q_{1} \vee{ }_{2} Q_{2}\right)\right\},-\infty<i<\infty$, are independent and ${ }_{2} Q_{1}$ and ${ }_{2} Q_{2}$ are independent,

(9) $\quad d\left({ }_{2} Q_{1} \vee{ }_{2} Q_{2}\right)=d\left({ }_{1} Q_{1} \vee{ }_{1} Q_{2}\right)$,

(10) $\quad P_{2} \mathcal{E}_{2} \bigvee_{-K_{2}}^{K_{2}} T^{i}\left({ }_{2} Q_{1} \bigvee_{2} Q_{2}\right)$,

(11) $\quad D\left({ }_{2} Q_{1} \vee_{2} Q_{2},{ }_{1} Q_{1} \vee_{1} Q_{2}\right)<\varepsilon_{2}$

Hence by the choice of $\varepsilon_{2}$, (11) implies

(12) $\quad P_{1}{ }^{2^{-1}} \bigvee_{-K_{1}}^{K_{1}} T^{i}{ }_{2} Q_{1}$

Applying Lemma 15 for $P_{3}, T$ and ${ }_{2} Q_{1} \vee_{2} Q_{2}$, we have a partition ${ }_{2} Q_{3} \subset \bigvee_{-\infty}^{\infty} T^{i} P_{3}$ such that

(13) $H\left({ }_{2} Q_{1}\right)+H\left({ }_{2} Q_{2}\right)+H\left({ }_{2} Q_{3}\right)=h\left(P_{3}, T\right)$,

(14) $\left\{T^{i}\left(\stackrel{3}{\vee}_{1} Q_{j}\right)\right\},-\infty<i<\infty$, are independent and $\left\{{ }_{2} Q_{j}\right\}, 1 \leqq j \leqq 3$, are independent.

Similarly we get a sequence of partitions $\left\{{ }_{i} Q_{j} ; 1 \leqq j \leqq i+1, i \geqq 1\right\}$ and 
a sequence of positive integers $\left\{K_{i}\right\}, i \geqq 1$, by induction such that

$$
\bigvee_{1}^{n} Q_{j} \subset \bigvee_{-\infty}^{\infty} T^{i} P_{n} ;{ }_{n} Q_{n+1} \subset \bigvee_{-\infty}^{\infty} T^{i} P_{n+1}
$$

(16) $\left\{T^{i}\left(\bigvee_{1}^{n+1}{ }_{n} Q_{j}\right)\right\},-\infty<i<\infty$, are independent,

(17) $\left\{{ }_{n} Q_{j}\right\}, 1 \leqq j \leqq n+1$, are independent,

(18) $\quad d\left({\underset{1}{V}}_{n}^{n} Q_{j}\right)=d\left(\bigvee_{1}^{n}{ }_{n-1} Q_{j}\right)$

(19) $\quad P_{m} 2^{2^{-m}+\cdots+2^{-n}} \bigvee_{-K_{m}}^{K_{m}} T^{i}\left(\bigvee_{1}^{m}{ }_{n} Q_{j}\right), \quad 1 \leqq m \leqq n$,

(20) $\quad D\left(\bigvee_{1}^{n}{ }_{n} Q_{j}, \stackrel{n}{\vee}_{1}^{n}{ }_{n-1} Q_{j}\right)<2^{-n}$,

(21) $\sum_{1}^{n+1} H\left({ }_{n} Q_{j}\right)=h\left(P_{n+1}, T\right)$.

By (20) we obtain partitions ${ }_{\infty} Q_{j}, j \geqq 1$, such that

$$
D\left({ }_{n} Q_{j},{ }_{\alpha} Q_{j}\right) \rightarrow 0, \quad \text { as } n \rightarrow \infty .
$$

Then $d\left({ }_{\alpha} Q_{j}\right)=d\left({ }_{j} Q_{j}\right)=d\left({ }_{j-1} Q_{j}\right),\left\{{ }_{\alpha} Q_{j}\right\}, j \geqq 1$, are independent by (17), $\left\{T^{i}\left(\bigvee_{1}^{\infty}{ }_{\alpha} Q_{j}\right)\right\},-\infty<i<\infty$, are independent by (16), and by (19) we have

$$
P_{k}{ }^{2-k+1} \bigvee_{-\infty}^{\infty} T^{i}\left(\bigvee_{1}^{k} Q_{j}\right), \quad k=1,2, \ldots
$$

and so

$$
\bigvee_{-\infty}^{\infty} T^{i}\left({\underset{1}{\vee}}_{\infty}^{\infty} Q_{j}\right)={\underset{-\infty}{\vee}}^{\infty} T^{i} P
$$

Thus $R=\bigvee_{1}^{\infty} Q_{j}$ is a Bernoulli generator for $T$ and so $(R, T)$ is a Bernoulli shift.

Now we will prove the isomorphism between the generalized weak Bernoulli transformations $T_{1}$ and $T_{2}$ with $h\left(T_{1}\right)=h\left(T_{2}\right) \leqq \infty$. Using the above argument there exist two partitions $R_{1}$ and $R_{2}$ such that $\left(R_{1}, T_{1}\right)$ and $\left(R_{2}, T_{2}\right)$ are Bernoulli shifts. If $h\left(T_{1}\right)=h\left(T_{2}\right)<\infty$, then $H\left(R_{1}\right)=$ $H\left(R_{2}\right)<\infty$ and therefore $R_{1}$ and $R_{2}$ are at most countable partitions. So 
that $T_{1}$ and $T_{2}$ are isomorphic by Proposition 1 .

When $h\left(T_{1}\right)=h\left(T_{2}\right)=\infty$, we will prove that they are isomorphic to the Bernoulli shift on the product space $\prod_{-\infty<i<\infty} X_{i}, X_{i}=[0,1]$. Let $P$ be a generalized weak Bernoulli generator for $T$ and $h(T)=h(P, T)=\infty$. Then we can also assume in the above argument that $h\left(P_{n}, T\right)<h\left(P_{n+1}, T\right)$ $-\log 2, n \geqq 1$ (by choosing subsequence of $P_{n}$ ). Noting that the condition for the choice of $\pi$ in the proof of Lemma 15 is only $H(\pi)=h(T)-H(R)$, we can assume that all atoms of ${ }_{n} Q_{n-1}$ have measures less than $1 / 2$. Hence for the partition $R$ taken for $P$ and $T$ in the above argument, the factor space $X / R$ is isomorphic to the space $[0,1]$ with the ordinary Lebesgue measure. Thus the proof of the theorem is complete.

\section{§5. Examples}

We will now give some examples of weak Bernoulli transformations. Firstly we will discuss Markov shifts. Let $X_{0}$ be $\{1,2, \ldots, N\}$ or $\{1,2, \ldots\}$ the set of all positive integers. Let $X=\prod_{-\infty}^{\infty} X_{n}$ where $X_{n}=X_{0}$ for all $n$. Let $M=\left(m_{i j}\right)_{i, j \in X_{0}}$ be a transition matrix with stationary probability $\left\{m_{i} ; i \in X_{0}\right\}$;

$$
\sum_{i \in X_{0}} m_{i} m_{i j}=m_{j}, \quad j \in X_{0} .
$$

We assume $m_{i}>0$ for all $i \in X_{0}$. The pair of $M$ and $\left\{m_{i}\right\}$ gives a Markov measure $m$ on $\mathscr{F}$, where $\mathscr{F}$ is the complete $\sigma$-field generated by cylinders. The shift $T$ on $X$ defined by

$$
(T x)_{n}=x_{n-1}, \quad n=0, \pm 1, \ldots, \quad x=\left(\ldots, x_{0}, x_{1}, \ldots\right) \in X
$$

preserves the measure $m ; T$ on $(X, \mathscr{F}, m)$ is called a Markov shift. We assume $T$ is ergodic. Thus $M$ is assumed to be irreducible, recurrent and of positive type. Let $M^{k}=\left(m_{i j}^{(k)}\right)$ be the $k$-step transition matrix. It is easy to see that $T$ is mixing if and only if

(1) $\lim _{k \rightarrow \infty} m_{i j}^{(k)}=m_{j}, \quad i, j \in X_{0}$.

Note (1) holds if and only if $M$ is aperiodic. 
Let $P=\left\{p_{j} ; j \in X_{0}\right\}$ be the partition of $X$ defined as $p_{j}=\left\{x ; x_{0}=j\right\}$, $j \in X_{0}$. Denote $P_{j}^{n}=\bigvee_{j}^{n} T^{i} P$ for $j \leqq n$. We will prove the following

Proposition 2. $P$ is weak Bernoulli for $T$ if and only if (1) holds.

First we will see that $P$ is a $\mathrm{K}$-partition for $T$, i.e. $\bigcap_{n} P_{-\infty}^{n}$ is trivial, if and only if (1) holds. Indeed $P$ is a $\mathrm{K}$-partition for $T$ if and only if

(2) $\lim _{k \rightarrow \infty} \sum_{i}\left|\sum_{j} f(j) m_{i j}^{(k)}-\sum_{j} f(j) m_{j}\right| m_{i}=0$

for every bounded function $f$ on $X_{0}$ (cf. [8]), and it is easy to see that (2) is equivalent to (1). On the other hand the Markov property implies for each $n \geqq 0$

$$
\begin{aligned}
& \quad \sum_{p \in P_{-n}^{0}, q \in P_{k}^{k+n}}|m(p \cap q)-m(p) m(q)| \\
& =\sum_{i, j \in X_{0}}\left|m_{i} m_{i j}^{(k)}-m_{j}\right| \\
& =2 \sum_{i \in X_{0}} m_{i} \sup _{J \subset X_{0}}\left|\sum_{j \in J} m_{i j}^{(k)}-\sum_{j \in J} m_{j}\right| .
\end{aligned}
$$

Since $k$-step transition probabilities of the time reversed chain are $\hat{m}_{i j}^{(k)}=$ $m_{j} m_{j i}^{(k)} / m_{i}, i, j \in X_{0}$, if (1) holds then $P$ is also a K-partition for $T^{-1}$ and hence

(4) $\lim _{k \rightarrow \infty} \sup _{B \in P_{k}^{\infty}}|m(A \cap B)-m(A) m(B)|=0$

for each $A \in \mathscr{F}$. Take $A=\left\{x_{0}=i\right\}$ and $B=\left\{x_{k} \in J\right\}$, then (4) implies (3) converges to 0 uniformly in $n$ as $k \rightarrow \infty$. Thus mixing Markov shifts with countable states are weak Bernoulli transformations.

We will now consider the continued-fraction transformation $T$ on $X=$ $[0,1]$ defined by

$$
T x=\left\{\begin{array}{cc}
\{1 / x\}, & x \neq 0 \\
0, & x=0
\end{array}\right.
$$

with the invariant measure 


$$
m(A)=\frac{1}{\log 2} \int_{A} \frac{d x}{1+x}, \quad A \in \mathscr{F} .
$$

We will assert that $T$ is weak Bernoulli, although it is not invertible. Consider the partition $P=\{[1 /(j+1), 1 / j] ; j=1,2, \ldots\}$ of $X$. Let $P_{j}^{n}=\bigvee_{j}^{n} T^{-i} P$ for $0 \leqq j \leqq n$. It is easy to see that $P$ is a generator. Let $\Delta_{n}$ be the generic atom of $P_{0}^{n}$. Then it is known that

$$
m \cdot\left(T^{-(k+n)} A \mid \Delta_{n}\right)=m(A)\left(1+\theta \rho^{k}\right), \quad A \in \mathscr{F},
$$

where $|\theta| \leqq M, \rho<1$ and $M$ and $\rho$ are constants independent of $A, k, n$, and $\Delta_{n}$ (cf. $[1]$, p. 50). This implies

$$
\sum_{\Delta_{n} \in P_{0}^{n}, \Delta \in P_{n+k}^{2 n+k}}\left|m\left(\Delta_{n} \cap \Delta\right)-m\left(\Delta_{n}\right) m(\Delta)\right| \leqq M \rho^{k} .
$$

Thus $T$ is weak Bernoulli with the generator $P$, and hence each natural extension of $T$ is a Bernoulli transformation.

We remark that Y. Takahashi and one of the authors $[3]$ showed that $\beta$-expansion transformations are also weak Bernoulli, while they are not invertible.

\section{References}

[1] Billingsley, P., Ergodic Theory and Information, Wiley, New York, 1965.

[2] Friedman N. A. and D. S. Ornstein, On isomorphism of weak Bernoulli transformations, Advances in Math. 5 (1971), 365-394.

[3] Ito, S. and Y. Takahashi, Markov subshifts, to appear.

[4] Ornstein, D.S., Bernoulli shifts with the same entropy are isomorphic, Advances in Math. 4 (1970), 337-352.

[5] - Two Bernoulli shifts with infinite entropy are isomorphic, Advances in Math. 5 (1971), 339-348.

[6] Rohlin, V.A., On the fundamental ideas of measure theory, Mat. Sb. 25 (67) (1949), 107-150. [Amer. Math. Soc. Transl. I. Ser. 10 (1961), 1-54.]

[7] Smorodinsky, M., An exposition of Ornstein isomorphism theorem, to appear.

[8] Totoki, H., On a class of special flows, Z. Wahrscheinlichkeitstheorie und Verw. Gebiete, 15 (1970), 157-167. 